\title{
Conjectures on the Quotient Ring by Diagonal Invariants
}

\author{
MARK D. HAIMAN \\ Department of Mathematics, University of Califormia, San Diego, La Jolla, CA 92093-0112.
}

Received February 3, 1993; Revised July 7, 1993

\begin{abstract}
We formulate a series of conjectures (and a few theorems) on the quotient of the polynomial ring $\mathbb{Q}\left[x_{1}, \ldots, x_{n}, y_{1}, \ldots, y_{n}\right]$ in two sets of variables by the ideal generated by all $S_{n}$ invariant polynomials without constant term. The theory of the corresponding ring in a single set of variables $X=\left\{x_{1}, \ldots, x_{n}\right\}$ is classical. Introducing the second set of variables leads to a ring about which little is yet understood, but for which there is strong evidence of deep connections with many fundamental results of enumerative combinatorics, as well as with algebraic geometry and Lie theory.
\end{abstract}

Keywords: diagonal harmonics, invariant, Coxeter group

\section{Introduction}

It has recently been discovered, mainly on the basis of evidence obtained using the computer algebra system MACAULAY, that there seem to be unexpected and profound connections between a certain natural ring and some fundamental and much-studied aspects of combinatorics and algebraic geometry. The ring in question is the quotient of the polynomial ring $\mathbb{Q}\left[x_{1}, \ldots, x_{n}, y_{1}, \ldots, y_{n}\right]$ by the ideal generated by all $S_{n}$ invariant polynomials without constant term. This paper is an attempt to treat in a reasonably comprehensive way a series of conjectures (and a few theorems) concerning the structure of this ring as a doubly graded $S_{n}$ module. Besides listing the existing conjectures and the current state of knowledge about them, I have tried, especially in the later sections, to outline some of their combinatorial and geometric implications.

A number of people were involved in formulating these conjectures and exploring various observations about them related here. I have made some attempt to give credit through notes appended to many sections. I hope I have not inadvertently shortchanged any of the many contributors to this work.

\section{Essential definitions}

\section{1. $S_{n}$ action on $\mathbb{Q}\left[x_{1}, \ldots, x_{n}, y_{1}, \ldots, y_{n}\right]$}

We will be concerned with the diagonal action of the permutation group $S_{n}$ by automorphisms of the polynomial ring $\mathbb{Q}[X, Y]=\mathbb{Q}\left[x_{1}, \ldots, x_{n}, y_{1}, \ldots, y_{n}\right]$ in 
two sets of variables $X$ and $Y$. By the diagonal action we mean the one given by $\sigma\left(x_{i}\right)=x_{\sigma(i)}, \sigma\left(y_{i}\right)=y_{\sigma(i)}$ for $\sigma \in S_{n}$. We call this action diagonal because it results from combining the double action of $S_{n} \times S_{n}$ permuting the $X$ and $Y$ variables separately with the diagonal embedding of $S_{n}$ into $S_{n} \times S_{n}$ given by $\sigma \mapsto(\sigma, \sigma)$.

We may think of the variables $X$ and $Y$ as coordinates on a $2 n$-dimensional vector space $V$, and regard group actions on $\mathbb{Q}[X, Y]$ as arising from actions on $V$.

The double action of $S_{n} \times S_{n}$ on $V$ is a reflection group action: When $\tau$ is a transposition $(i j)$ the elements $(\tau, 1)$ and $(1, \tau)$ act on $V$ by exchanging two coordinates and so are reflections. That is, each of them fixes a hyperplane of codimension 1 and acts as -1 on a complementary 1-dimensional subspace. Since these elements generate $S_{n} \times S_{n}$, the action is, by definition, a reflection group action.

The diagonal action of $S_{n}$ on $V$ by contrast is not a reflection group action. In fact, every element of $S_{n}$ acts by a transformation with determinant 1 on $V$, whereas the determinant of a reflection is -1 , so there are no reflections in this action at all.

We may decompose $V$ as $V=U \oplus W$, where $U$ and $W$ are the subspaces defined by $X=0$, and $Y=0$, respectively. Then $U$ and $W$ each carry the natural reflection group action of $S_{n}$. From this point of view, the diagonal action of $S_{n}$ is a special case of the action of a reflection group $W$ on the coordinate ring $\mathbb{Q}[U \oplus U]$, where $U$ carries the reflection action. As we shall see, the attempt to generalize the conjectures made here to this setting leads to some interesting phenomena.

From the standpoint of reflection groups, it would seem sensible to use the irreducible reflection group action $U$ of $S_{n}$, which in coordinates is the action on the space spanned by $\left\{x_{1}-x_{2}, x_{2}-x_{3}, \ldots, x_{n-1}-x_{n}\right\}$. However, the object of our study will be a quotient ring by $S_{n}$ invariants of positive degree, so we will always be "modding out" the invariant $x_{1}+\cdots+x_{n}$. As a result we lose nothing and gain convenience of notation by working with $\mathbb{Q}[X, Y]$ instead of $\mathbb{Q}[U \oplus U]$.

\subsection{Ideal $I$ and quotient ring $R_{n}$}

We now define the ring $R_{n}$, properties of which are described by our conjectures.

Let $I$ be the ideal in $\mathbb{Q}[X, Y]$ generated by all $S_{n}$ invariant polynomials without constant term. We set

$$
R_{n}=\mathbb{Q}[X, Y] / I \text {. }
$$

Note that $I$ is a homogeneous ideal, since if $p(X, Y)$ is an invariant polynomial without constant term, then so is each of its homogeneous components of various degrees. In fact, the same is true for homogeneous components of various bidegrees, where we say that a polynomial has bidegree $(i, j)$ if it has degree 
$i$ in $X$ and $j$ in $Y$. Thus $I$ is a bihomogeneous ideal, and consequently $R_{n}$ has the structure of a doubly graded ring. In other words, $R_{n}$ is the direct sum of homogeneous subspaces $\left(R_{n}\right)_{i, j}$, where $\left(R_{n}\right)_{i, j}$ consists of all images of polynomials $p(X, Y)$ which are homogeneous of bidegree $(i, j)$. It is immediate that $\left(R_{n}\right)_{i, j}\left(R_{n}\right)_{i^{\prime}, j^{\prime}} \subseteq\left(R_{n}\right)_{i+i^{\prime}, j+j^{\prime}}$.

The ideal $I$ is an $S_{n}$ submodule of the ring $\mathbb{Q}[X, Y]$, so the quotient ring $R_{n}$ acquires an $S_{n}$ action. The action preserves bidegree, so the decomposition $R_{n}=\oplus_{i, j}\left(R_{n}\right)_{i, j}$ is a decomposition into $S_{n}$ submodules. All the conjectures propose answers to the question, what are the characters of these submodules? At present there is no conjecture giving a complete answer. Instead, there are conjectures for various marginals of the matrix of characters, which are seen to be full of wonderful combinatorial surprises.

It is easy to show that any set of homogeneous generators for the subring of invariants $\mathbb{Q}[X, Y]^{S_{n}}$ generates the ideal $I$, and conversely. The corresponding statement in fact holds for any finite group action. In this particular case, the following theorem of Weyl gives such generators.

Proposition 1.2.1 (Weyl [29]). The ring of invariants $\mathbb{Q}[X, Y]^{S_{n}}$ is generated by the "polarized power sums" $p_{r, s}(X, Y)=\sum_{i} x_{i}^{r} y_{i}^{s}$.

This result generalizes to any number of sets of variables $X, Y, Z, \ldots$, and has an analog for the signed permutation group $B_{n}$, but not for the other classical Weyl groups $D_{n}$.

We make a trivial observation here whose significance will develop later.

PROPOSITION 1.2.2. The homogeneous subspace $\left(R_{n}\right)_{0,0}$ affords the trivial representation of $S_{n}$ and this is the only occurrence of the trivial representation in $R_{n}$.

Proof. Obviously $\left(R_{n}\right)_{0,0}$ affords the trivial representation, since it is spanned by 1. If $(i, j) \neq(0,0)$, then every $S_{n}$ invariant polynomial homogeneous of bidegree $(i, j)$ belongs to $I$ by definition, so there are no $S_{n}$ invariants in $\left(R_{n}\right)_{i, j}$.

\subsection{Diagonal harmonics $H_{n}$}

There is a useful alternative view of $R_{n}$ as isomorphic to a space of harmonics, as we now explain.

Definition. The apolar form (to use Rota's terminology) is the nondegenerate symmetric bilinear form $\langle\cdot, \cdot\rangle$ on $\mathbb{Q}[X, Y]$ defined by

$$
\langle f, g\rangle=\left.f\left(\partial x_{1}, \ldots, \partial x_{n}, \partial y_{1}, \ldots, \partial y_{n}\right) g\left(x_{1}, \ldots, x_{n}, y_{1}, \ldots, y_{n}\right)\right|_{X=Y=0},
$$

where $\partial z$ denotes the partial derivative operator with respect to $z$. 
To see that the apolar form is symmetric and nondegenerate, merely observe that the set of all monomials in $X, Y$ forms an orthogonal basis for it. Note also that the bihomogeneous subspaces $(\mathbb{Q}[X, Y])_{i, j}$ are all mutually orthogonal, and that $\langle\cdot, \cdot\rangle$ restricts to a nondegenerate form on each of them. Consequently, provided we deal with homogenous subspaces, we may generally treat the apolar form as if it were defined on a finite dimensional vector space, so that we have $I^{\perp \perp}=I$, for instance, when $I$ is homogeneous.

PROPOSITION 1.3.1. If $I$ is a homogeneous ideal, then its orthogonal complement $H=I^{\perp}$ is a homogeneous space of polynomials, closed under the taking of arbitrary partial derivatives. We also have $H=\{h \mid f(\partial X, \partial Y) h=0 \forall f \in I\}$; in other words, regarding $I$ as a system of polynomial partial differential equations, $H$ is its space of solutions. If $H$ is any homogeneous space of polynomials closed under partial derivatives, then $I=H^{\perp}$ is a homogeneous ideal with $H=I^{\perp}$.

Proof. From the definition above of $\langle\cdot, \cdot\rangle$, we see immediately that

$$
\langle f, \partial x g\rangle=\langle x f, g\rangle,
$$

that is, the operator $\partial x$ is adjoint to multiplication by $x$. Here $x$ is any variable from $X$ or $Y$.

In particular, if $h \in I^{\perp}$, then $\langle f, h\rangle=0$ for all $f \in I$, and hence $\langle x f, h\rangle=$ $\langle f, \partial x h\rangle=0$, since $I$ is an ideal. Thus $\partial x h \in I^{\perp}$. This shows $H=I^{\perp}$ is closed under derivatives.

To show $H=\{h \mid f(\partial X, \partial Y) h=0 \forall f \in I\}$, note first that any element of this set obviously belongs to $I^{\perp}$. Conversely, if $h \in I^{\perp}$, then by definition $\left.f(\partial X, \partial Y) h\right|_{X=Y=0}=0$ for all $f \in I$. Replacing $f$ by an arbitrary multiple of itself, we see that the partial derivatives of all orders of $f(\partial X, \partial Y) h$ vanish at 0 , and therefore the polynomial $f(\partial X, \partial Y) h$ vanishes identically.

Finally, let $H$ be an arbitrary homogeneous space of polynomials closed under differentiation. If $f \in H^{\perp}$, then $\langle x f, h\rangle=\langle f, \partial x h\rangle=0$ for all $h \in H$. This shows $I=H^{\perp}$ is an ideal, and $H=H^{\perp \perp}=I^{\perp}$ follows because the spaces are homogeneous.

Remark. Homogeneity was used only in the last sentence of the proof. The proposition can be generalized to inhomogeneous ideals if we are willing to admit a space of formal power series for $H$.

Definition. The space $H_{n}$ of diagonal harmonics for $S_{n}$ is $I^{\perp}$, where $I$ is the ideal defining the ring $R_{n}$ in (1).

Since the apolar form is $S_{n}$ invariant, the space $H_{n}$ is an $S_{n}$ submodule of $\mathbb{Q}[X, Y]$, as is each of its homogeneous components $\left(H_{n}\right)_{i, j}$. As $S_{n}$ module, $\left(H_{n}\right)_{i, j}$ is clearly isomorphic to $\left(R_{n}\right)_{i, j}$, since both are complements to $(I)_{i, j}$ in 
$(\mathbb{Q}[X, Y])_{i, j}$. An explicit isomorphism $\alpha: H_{n} \rightarrow R_{n}$ can be given by simply letting $\alpha(h)$ be the element of $R_{n}$ represented modulo $I$ by the polynomial $h$.

Harmonics can be defined in more generality than we have just done. Namely, let $V$ be a finite-dimensional vector space equipped with a nondegenerate symmetric bilinear form $\langle\cdot, \cdot\rangle$, and let $X=\left\{x_{1}, \ldots, x_{n}\right\}$ be a basis of coordinates on $V$. There is then a unique dual basis of operators $\partial u_{1}, \ldots, \partial u_{n}$ on $\mathbb{Q}[X]$, where the $\partial u_{i}$ are linear combinations of $\partial x_{j}$ chosen so that $\partial u_{i} x_{j}=\left\langle x_{i}, x_{j}\right\rangle$. The form $\langle\cdot, \cdot\rangle$ may now be extended to all of $\mathbb{Q}[X]$ by a formula like (2), and the result is independent of the choice of basis.

In particular, if the original form $\langle\cdot, \cdot\rangle$ is invariant under the action of a group $G$ on $V$ then the extended form is invariant for the induced action on $\mathbb{Q}[X]$. Proposition 1.3.1 still applies, and we obtain a space of " $G$-harmonics" orthogonal to the ideal generated by $G$ invariants without constant term.

All the (real) reflection groups have reflection representations defined over $\mathbb{Q}$ and leaving invariant a suitable form $\langle\cdot, \cdot\rangle$, so we can construct harmonics for their reflection actions and their diagonal actions, just as for $S_{n}$.

Our use of $\mathbb{Q}$ as a ground field is, incidentally, quite arbitrary. Everything works identically over $\mathbb{R}$, and can be adapted to Hermitian forms on complex vector spaces by using antiholomorphic partial derivatives.

Notes. The notion of harmonics considered here is standard in harmonic analysis on groups, see e.g. [12]. A. Garsia was the first to point out the value of studying $R_{n}$ via the diagonal harmonics. The advantages resulting from this perspective will become evident in Section 5.

\subsection{Hilbert series and Frobenius series}

Whenever we deal with graded spaces, such as the polynomial ring $\mathbb{Q}[X, Y]$ or any homogeneous ideal, quotient ring, or space of harmonics, it is convenient to keep track of the dimensions of its homogeneous components by means of a generating function, known as the Hilbert series.

When our space is graded by a single degree, say $A=(A)_{0} \oplus(A)_{1} \oplus \cdots$, we will write a Hilbert series in a single variable $q$, denoting it by the letter $\mathcal{H}$, as

$$
\mathcal{H}_{A}(q)=\sum_{i=0}^{\infty} q^{i} \operatorname{dim}(A)_{i} .
$$

More often, we will have bigraded spaced $A=(A)_{0,0} \oplus(A)_{0,1} \oplus(A)_{1,0} \oplus \cdots$, in which case we write a bivariate Hilbert series such as

$$
\mathcal{H}_{A}(t, q)=\sum_{i=0}^{\infty} \sum_{j=0}^{\infty} t^{i} q^{j} \operatorname{dim}(A)_{i, j}
$$


In dealing with graded $S_{n}$ modules we will generally want to record not only the dimensions of homogeneous components but their characters. To do this we combine the notion of Hilbert series with the familiar Frobenius map assigning to each character $\chi$ of $S_{n}$ a symmetric polynomial $\phi(\chi)$ so that the irreducible character $\chi_{\lambda}$ corresponds to the Schur function $s_{\lambda}$. (We assume familiarity with the theory of symmetric functions and their relationship to $S_{n}$ characters, as developed for instance in [17].)

Thus to a bigraded $S_{n}$ module $A=(A)_{0,0} \oplus(A)_{0,1} \oplus(A)_{1,0} \oplus \cdots$ we attach a Frobenius series

$$
\mathcal{F}_{A}(t, q)=\sum_{i=0}^{\infty} \sum_{j=0}^{\infty} t^{i} q^{j} \phi\left((A)_{i, j}\right),
$$

where by abuse of notation $\phi$ of a module means $\phi$ of its character. The Frobenius series is a symmetric function (in some infinite alphabet $Z$ which we leave unspecified), with coefficients in the ring of polynomials $\mathbb{Z}[t, q]$.

Clearly the Frobenius series determines the Hilbert series. To make this explicit, note that the degree of the $S_{n}$ character $\chi_{\lambda}$ is given by $\left\langle p_{1}^{n}, s_{\lambda}\right\rangle$, where $\langle\cdot, \cdot\rangle$ is here the usual inner product on symmetric functions and $p_{k}$ is the $k$ th power sum. Therefore we may write

$$
\mathcal{H}_{A}(t, q)=\left\langle p_{1}^{n}, \mathcal{F}_{A}(t, q)\right\rangle .
$$

For example, by a well-known computation based on the master theorem of MacMahon, we have for $\mathbb{Q}[X]$,

$$
\mathcal{F}(q)=\sum_{|\lambda|=n} s_{\lambda}\left(1, q, q^{2}, \ldots\right) s_{\lambda}=\left.\prod_{i, j} \frac{1}{1-q^{i} z_{j} u}\right|_{u^{n}} ; \mathcal{H}(q)=\frac{1}{(1-q)^{n}}
$$

Here $\left\{z_{1}, z_{2}, \ldots\right\}$ is the alphabet of variables in which the symmetric polynomials are taken.

For the bigraded ring $\mathbb{Q}[X, Y]$ we have

$$
\begin{aligned}
\mathcal{F}(t, q) & =\sum_{|\lambda|=n} \sum_{|\mu|=n} s_{\lambda}\left(1, t, t^{2}, \ldots\right) s_{\mu}\left(1, q, q^{2}, \ldots\right) s_{\lambda} * s_{\mu} \\
& =\left.\prod_{i, j, k} \frac{1}{1-t^{i} q^{j} z_{k} u}\right|_{u^{n}}
\end{aligned}
$$

and

$$
\mathcal{H}(t, q)=\frac{1}{(1-t)^{n}(1-q)^{n}} .
$$

Here $s_{\lambda} * s_{\mu}$ is the internal product $\phi\left(\chi_{\lambda} \otimes \chi_{\mu}\right)$. 


\subsection{The case of one set of variables for comparison}

In this section, we consider the action of $S_{n}$ on $\mathbb{Q}[X]$, where $X=\left\{x_{1}, \ldots, x_{n}\right\}$, and the analogs of $R_{n}$ and $H_{n}$. Everything we say holds, when suitably restated, for every reflection group, and nearly all of the properties we discuss are actually characteristic of reflection groups, in that if any one of them holds for a finite group of linear transformations, then it must be generated by reflections. This theory is treated in the classical papers of Chevalley, Shephard, Todd, and Steinberg $[4,21,24,25,26]$.

In the present situation, we consider the ideal $I$ generated by all invariant polynomials in $X$ without constant term, or equivalently by any set of generators for the ring of symmetric polynomials in $X$, such as the power sums $p_{1}(X), \ldots, p_{n}(X)$.

These generators form a homogeneous system of parameters (abbreviated h.s.o.p.) in $\mathbb{Q}[X]$, that is, a set of $n$ homogeneous and nonconstant polynomials $p_{i}$ such that $\mathbb{Q}[X] /\left(p_{1}, \ldots, p_{n}\right)$ is a 0 -dimensional ring (or finite-dimensional vector space). The elements of an h.s.o.p. are algebraically independent, so $\mathbb{Q}[X]^{S_{n}}$ is isomorphic to the polynomial ring $\mathbb{Q}\left[p_{1}, \ldots, p_{n}\right]$, and the full ring $\mathbb{Q}[X]$ is a free module over this subring.

For this section only, we put $R_{n}=\mathbb{Q}[X] / I$ and $H_{n}=I^{\perp}$, the one set of variables versions of the quotient ring and harmonics which we defined earlier for $X$ and $Y$.

The space $H_{n}$, or indeed any space of polynomials mapping isomorphically onto $R_{n}$ modulo $I$, generates $\mathbb{Q}[X]$ as a free module over $\mathbb{Q}[X]^{S_{n}}$, which immediately gives the equation

$$
\frac{\mathcal{F}(q)}{(1-q) \cdots\left(1-q^{n}\right)}=\sum_{|\lambda|=n} s_{\lambda}\left(1, q, q^{2}, \ldots\right) s_{\lambda}
$$

for the Frobenius series $\mathcal{F}(q)$ of $H_{n}$ (and $R_{n}$ ). This completely solves the character problem: The polynomial whose coefficients give the multiplicities of $\chi_{\lambda}$ in the various components $\left(R_{n}\right)_{i}$ is

$$
(1-q) \cdots\left(1-q^{n}\right) s_{\lambda}\left(1, q, q^{2}, \ldots\right)
$$

which by the theory of symmetric functions is also equal to

$$
q^{n(\lambda)} \frac{[n]_{q}[n-1]_{q} \cdots[1]_{q}}{\prod_{x \in \lambda}[h(x)]_{q}}=\sum_{T \in S Y T(\lambda)} q^{\operatorname{maj}(T)}
$$

where $[k]_{q}$ means $1+q+\cdots+q^{k-1} ; h(x)$ is the length of the "hook" of a cell $x$ in the diagram of $\lambda$; the sum ranges over standard Young tableaux of shape $\lambda$; and $n(\lambda)$ and maj $(T)$ are certain statistics associated with $\lambda$ and $T$. 
It also follows immediately from our free module situation that the Hilbert series of $H_{n}$ (and $R_{n}$ ) is

$$
[n]_{q} !=[n]_{q}[n-1]_{q} \cdots[1]_{q},
$$

and consequently that $\operatorname{dim}_{\mathbb{Q}}\left(H_{n}\right)=\operatorname{dim}_{\mathbb{Q}}\left(R_{n}\right)=n !$, the order of the group $S_{n}$. In fact, this space affords the regular representation of $S_{n}$, as can be seen from (13).

The space $H_{n}$ in this case has a simple direct description. It contains the Vandermonde determinant $\Delta(X)=\prod_{i<j}\left(x_{i}-x_{j}\right)$, and consists exactly of this polynomial and its partial derivatives of all orders. For other reflection groups $\Delta(X)$ would be replaced by the discriminant, the product of linear forms vanishing along the reflecting hyperplanes of all reflections in the group.

\section{The main conjectures, roughly in order of increasing strength}

In this section we present a series of conjectures about the Hilbert series and Frobenius series of $R_{n}$, or equivalently, of $H_{n}$. Using the computer algebra system MACAULAY, the conjectures on the Hilbert series have been checked through $n=7$ and the conjectures on the Frobenius series through $n=6$. Tables appear in the appendix.

\section{1. $(n+1)^{n-1}$}

The first (and earliest) conjecture concerns simply the vector space dimension of $R_{n}$ and $H_{n}$, or $\mathcal{H}_{n}(1,1)$ in Hilbert series notation.

Conjecture 2.1.1

$$
\mathcal{H}_{n}(1,1)=(n+1)^{n-1} .
$$

Combinatorialists will recognize this quantity immediately as the number of rooted forests on $n$ labeled vertices, or equivalently as the number of unrooted trees on $n+1$ labeled vertices. It has other interpretations, which will develop below.

\section{2. $[n+1]_{q}^{(n-1)}$}

The conjectures in this section and the next concern certain "marginals" of the Hilbert series. It is convenient to picture the coefficients of $\mathcal{H}_{n}(t, q)$ as entries of an array, as shown here for $n=3$ and in the appendix for all $n \leq 7$. 


$$
\begin{array}{c|cccc}
t^{3} & 1 & & & \\
t^{2} & 2 & 1 & & \\
t^{1} & 2 & 3 & 1 & \\
t_{0} & 1 & 2 & 2 & 1 \\
\hline & q^{0} & q^{1} & q^{2} & q^{3}
\end{array}
$$

The diagram expresses the fact that

$$
\mathcal{H}_{3}(t, q)=1+2 t+2 q+2 t^{2}+3 t q+2 q^{2}+t^{3}+t^{2} q+t q^{2}+q^{3}
$$

Various sums of parts of the diagram can be expressed as specializations of the Hilbert series. For instance for the leftmost column or bottom row we have the following.

PROPOSITION 2.2.1

$$
\mathcal{H}_{n}(q, 0)=\mathcal{H}_{n}(0, q)=[n]_{q} !
$$

Proof. We are considering the part of degree 0 in $Y$, say, which reduces to the Hilbert series (14) for the case of one set of variables.

Remark. For the same reason, the Frobenius series specialization $\mathcal{F}_{n}(q, 0)$ is given by (11-13).

We now consider the sums along "antidiagonals" leading upward and to the right in the diagram, which in the example (16) are

$$
\begin{array}{lllllll}
1 & 2 & 3 & 4 & 3 & 2 & 1 .
\end{array}
$$

Note that by Proposition 2.2.1, the extreme nonzero entries along the first row and column occur at $\left.t^{(}\right)$and $q^{\left(\frac{1}{2}\right)}$, corresponding to the harmonics $\Delta(X)$ and $\Delta(Y)$, the Vandermonde determinants.

\section{Conjecture 2.2.1}

$$
q^{\left(\frac{n}{2}\right)} \mathcal{H}_{n}\left(q^{-1}, q\right)=\left([n+1]_{q}\right)^{n-1}=\left(1+q+\cdots+q^{n}\right)^{n-1} .
$$

In particular, this conjecture requires that $\pm\left(\begin{array}{c}n \\ 2\end{array}\right)$ are the extremes of the difference (degree in $X$ ) - (degree in $Y$ ). This is a fact, a consequence of Corollary 3.2.1.

The difference of degrees statistic corresponding to the specialization $t=q^{-1}$ is the weight for the $\mathfrak{s l}_{2}$ action which will be discussed further in Section 3.1. From this point of view, Conjecture 2.1.1 asserts that $R_{n}$ is a specific $\mathfrak{s h}_{2}$ module. A word of warning - the formula (20) does not make $\mathcal{H}_{n}\left(q^{-1}, q\right)$ the character of 
the $(n-1)$-st tensor power of some irreducible $\mathfrak{s}_{2}$ module. Indeed its $(n-1)$-st root $q^{-n / 2}[n+1]_{q}$ is not a character at all for $n$ odd, and describes a module with two irreducible components, of highest weights $n / 2$ and $n / 2-1$, for $n$ even.

Notes. Conjecture 2.2.1 was discovered by R. Stanley.

\subsection{Inversion enumerator for trees}

Next we shall give a conjecture describing the sums along columns, or along rows, of the diagram (16), or in other words, describing the dimensions of homogeneous components of $R_{n}$ by degree in $X$ or $Y$ alone.

First we must define the inversion number for a labeled tree or forest.

Definition. Let $T$ be a spanning tree on the $n+1$ vertices $\{0,1, \ldots, n\}$. Regarding 0 as the root of the tree, we say that $\{i<j\} \subseteq\{1, \ldots, n\}$ is an inversion in $T$ if vertex $j$ is an ancestor of $i$, i.e., $j$ lies on the path connecting $i$ to the root 0 .

Alternatively, we may define an inversion in a rooted forest on $\{1, \ldots, n\}$ to be $\{i<j\}$ such that $i$ and $j$ belong to the same tree and $j$ is an ancestor of $i$; clearly the forest will have the same set of inversions as the tree formed by adjoining 0 and connecting it to the roots of the forest.

Conjecture 2.3.1. $\mathcal{H}_{n}(1, q)$ is the inversion enumerator for trees,

$$
\mathcal{H}_{n}(1, q)=\sum_{T} q^{\mathrm{inv}(T)}
$$

where $T$ ranges over all spanning trees on $\{0,1, \ldots, n\}$ and $\operatorname{inv}(T)$ denotes the number of inversions of $T$.

A number of interesting observations may be made about this conjecture. First of all, for $q=0$ it corresponds to the fact that there are $n$ ! trees with no inversions, or increasing trees. There are well-known bijections establishing this (see [23]). It also says that $q^{\left(\frac{n}{2}\right)}$ corresponds to the extreme degree and occurs with coefficient 1 , since the unique forest with every possible inversion is a single chain decreasing from the root. This fact will follow from Proposition 3.2.1.

There are other natural tree statistics with the same distribution as inv $(T)$. The most interesting is the external activity of Tutte [27], defined as follows. Fix any total ordering < on the set of edges of the complete graph $K_{n+1}$ with vertex set $\{0,1, \ldots, n\}$. Given a spanning tree $T$, an edge $e$ not in $T$ is said to be extermally active if it is less than all edges along the unique path in $T$ connecting the endpoints of $e$. The external activity $e(T)$ is by definition the number of externally active edges. 
More generally, the notion of external activity applies to bases of an arbitrary matroid, in this case the circuit matroid of the complete graph $K_{n+1}$. The fundamental result of Tutte is that

$$
\sum_{T} q^{e(T)}
$$

is the characteristic polynomial of the matroid, which does not depend on the choice of the ordering < among edges.

The polynomials (21) and (22) are identical because of a relationship between the inversion enumerator for trees and the enumerator for spanning subgraphs of $K_{n+1}$. Let $J_{n}(q)$ denote the inversion enumerator given by (21), and let

$$
C_{n}(q)=\sum_{G} q^{E(G)}
$$

where $G$ ranges over spanning subgraphs of $K_{n+1}$ and $E(G)$ is the number of edges in $G$. A spanning subgraph means a subset of the edges which connects all the vertices, that is, which contains a spanning tree. Then we have by [11, 18]

$$
C_{n}(q)=J_{n}(1+q)
$$

Since the same identity holds with $J_{n}(q)$ denoting the characteristic polynomial (22), (21) and (22) are equal. It is straightforward to write down the exponential generating function for the sequence $C_{n}(q)$, yielding the generating function for $J_{n}(q)$ as in [18].

Notes. Conjecture 2.3.1 was discovered by both Stanley and myself. I am indebted to I. Gessel for much of the information about the inversion enumerator mentioned here.

\subsection{The whole $S_{n}$ module $\left(\mathbb{Z}_{n+1}^{n} / \mathbb{Z}_{n+1}\right)$}

In this and the next few sections we turn from Hilbert series conjectures to their Frobenius series analogs, which are of course stronger, but not always as immediately accessible.

Our first goal will be to determine $\mathcal{F}_{n}(1,1)$, which is to say, to determine the whole of $R_{n}$ as an $S_{n}$ module, ignoring the grading. Our conjecture is that $R_{n}$ is the tensor product of a certain permutation representation by the sign representation. We will first state the conjecture at the representation level, then rephrase it in terms of $\mathcal{F}_{n}$.

Conjecture 2.4.1. Let $V_{n}$ be the following $S_{n}$ module. Begin with the finite abelian group $\mathbb{Z}_{n+1}^{n}$, where $\mathbb{Z}_{n+1}$ is the integers modulo $n+1$, with $S_{n}$ acting by 
permuting coordinates. The element $(1,1, \ldots, 1) \in \mathbb{Z}_{n+1}^{n}$ generates a subgroup isomorphic to $\mathbb{Z}_{n+1}$ whose elements are $S_{n}$ invariant, so that $S_{n}$ acts on the quotient group $\mathbb{Z}_{n+1}^{n} / \mathbb{Z}_{n+1}$. Taking the elements of the finite set $\mathbb{Z}_{n+1}^{n} / \mathbb{Z}_{n+1}$ as a $\mathbb{Q}$-basis we have a permutation representation of $S_{n}$, which is $V_{n}$. Then $R_{n}$ is isomorphic as $S_{n}$ module to $\varepsilon \otimes V_{n}$, where $\varepsilon$ is the sign representation.

Note that the cardinality of $\mathbb{Z}_{n+1}^{n} / \mathbb{Z}_{n+1}$ is $(n+1)^{n-1}$, in agreement with Conjecture 2.1.1.

Now let us find the character of $V_{n}$. To do so, we first work out the character of the permutation representation afforded by $\mathbb{Z}_{n+1}^{n}$, then divide by $n+1$. The group structure of $\mathbb{Z}_{n+1}^{n}$ has no relevance here, so we regard it as merely the set $\{0, \ldots, n\}^{n}$ of sequences $\left(a_{1}, \ldots, a_{n}\right)$ with $0 \leq a_{i} \leq n$.

Among these sequences, each $S_{n}$ orbit contains a unique element of the form $\left(a_{1} \leq a_{2} \leq \cdots \leq a_{n}\right)$, for which there are unique integers $\mu_{1}, \ldots, \mu_{k}$ such that $a_{1}=\cdots=a_{\mu_{1}}<a_{\mu_{1}+1}=\cdots=a_{\mu_{1}+\mu_{2}}<\cdots$. The stabilizer of the element $\left(a_{1} \leq a_{2} \leq \cdots \leq a_{n}\right)$ is the Young subgroup $S_{\mu_{1}} \times \cdots \times S_{\mu_{k}}$, and the Frobenius image of the permutation representation afforded by its orbit is the symmetric function $h_{\mu}=h_{\mu_{1}} \cdots h_{\mu_{k}}$.

A given sequence $\mu_{1}, \ldots, \mu_{k}$ with $\mu_{1}+\cdots+\mu_{k}=n$ will clearly arise $\left(\begin{array}{c}n+1 \\ k\end{array}\right)$ times among all orbits. We may group together $\mu$ 's representing the same partition $\lambda=\left(\lambda_{1} \geq \lambda_{2} \geq \cdots \geq \lambda_{k}\right)$ of $n$ by noting that the number of distinct such sequences $\mu$ is $\left(m_{\left.m_{1}(\lambda), m_{2}(\lambda), . . .\right)}\right.$, where $m_{i}(\lambda)$ denotes the multiplicity of $i$ as a part of $\lambda$.

Let us agree to regard each partition $\lambda$ of $n$ as having enough parts of 0 to make the total number of parts equal to $n+1$, that is, to define $m_{0}(\lambda)$ so that $m_{0}(\lambda)+m_{1}(\lambda)+\cdots+m_{n}(\lambda)=n+1$. Then we may express the number of orbits contributing $h_{\lambda}$ to the Frobenius image simply as $\left(\begin{array}{c}n+1 \\ m_{0}(\lambda), m_{1}(\lambda), \ldots, m_{n}(\lambda)\end{array}\right)$.

Adding everything up, dividing by $n+1$, and applying the symmetric function involution $\omega$ to account for the sign twist, we arrive at the following equivalent formulation of Conjecture 2.4.1.

Conjecture 2.4.2

$$
\mathcal{F}_{n}(1,1)=\sum_{|\lambda|=n} \frac{1}{n+1}\left(\begin{array}{c}
n+1 \\
m_{0}(\lambda), m_{1}(\lambda), \ldots, m_{n}(\lambda)
\end{array}\right) e_{\lambda}
$$

It is possible to express (25) in terms of Schur functions as follows. First note that the multinomial coefficient

$$
\left(\begin{array}{c}
n+1 \\
m_{0}(\lambda), m_{1}(\lambda), \ldots, m_{n}(\lambda)
\end{array}\right)
$$

is none other than $m_{\lambda}(1,1, \ldots, 1)$, where $m_{\lambda}$ denotes the monomial symmetric function, and the argument consists of $n+1$ ones. Applying the Cauchy formula 


$$
\sum_{\lambda} m_{\lambda}(X) h_{\lambda}(Y)=\prod_{i, j} \frac{1}{1-x_{i} y_{j}}=\sum_{\lambda} s_{\lambda}(X) s_{\lambda}(Y),
$$

we transform (25) into

$$
\omega \mathcal{F}_{n}(1,1)=\sum_{|\lambda|=n}=\frac{s_{\lambda}(1,1, \ldots, 1)}{n+1} s_{\lambda}
$$

or

$$
\mathcal{F}_{n}(1,1)=\sum_{|\lambda|=n} \frac{s_{\lambda^{\prime}}(1,1, \ldots, 1)}{n+1} s_{\lambda}
$$

where $\lambda^{\prime}$ denotes the conjugate partition, and the arguments of the Schur functions in the numerators consist of $n+1$ ones.

From (29) we can see that Conjecture 2.4 .2 predicts a multiplicity of 1 for the trivial character (corresponding to $s_{(n)}$ ) as it must to agree with Proposition 1.2.2.

\subsection{Character refinement of 2.2}

Conjecture 2.4.2, once expressed in the form (29), has an evident " $q$-analog," which matches the data for the Frobenius series version of Conjecture 2.2.1. More precisely, we have the following.

Conjecture 2.5.1

$$
q^{\left({ }_{2}\right)} \mathcal{F}_{n}\left(q^{-1}, q\right)=\sum_{|\lambda|=n} \frac{s_{\lambda^{\prime}}\left(1, q, \ldots, q^{n}\right)}{1+q+\cdots+q^{n}} s_{\lambda}
$$

It is not at all obvious that the coefficients

$$
\frac{s_{\lambda^{\prime}}\left(1, q, \ldots, q^{n}\right)}{1+q+\cdots+q^{n}}
$$

appearing in (30) are even polynomials, let alone polynomials with nonnegative coefficients. The potential difficulties may be appreciated by observing that the expression

$$
\frac{s_{\lambda}\left(1, q, \ldots, q^{p-1}\right)}{1+q+\cdots+q^{p-1}}
$$

for arbitrary $p$ is not always a polynomial.

In order to justify Conjecture 2.5 .1 as at least reasonable, we will now determine for which $p$ the expression (32) is a polynomial, and show that it has positive integer coefficients for those $p$. 
PROPOSITION 2.5.1. The following are equivalent:

(1) $\frac{\theta_{\lambda}\left(1, q_{1} \ldots, q^{p-1}\right)}{1+q+\cdots+q^{p-1}}$ is a polynomial for every partition $\lambda$ of $n$.

(2) $\frac{s_{(n)}\left(1, q, \ldots, q^{p-1}\right)}{1+q+\cdots+q^{p-1}}$ is a polynomial.

(3) $p$ is relatively prime to $n$.

Proof. We'll show (3) $\Rightarrow(1)$ and $(2) \Rightarrow(3)$, since $(1) \Rightarrow(2)$ is trivial.

In (1) we can replace $s_{\lambda}$ by $h_{\lambda}$ without affecting the result, since $\left\{s_{\lambda}\right\}$ and $\left\{h_{\lambda}\right\}$ are each bases for the symmetric functions of degree $n$. Regarding (2) note also that $s_{(n)}=h_{n}$.

It can be shown without difficulty that $h_{r}\left(1, q, \ldots, q^{p-1}\right)$ is equal to the $q$ binomial coefficient

$$
\left[\begin{array}{c}
p+r-1 \\
r
\end{array}\right]_{q}=\frac{[p]_{q} \cdots[p+r-1]_{q}}{[r]_{q} !}
$$

The roots of the polynomial $1+q+\cdots+q^{p-1}$ are the $p$ th roots of unity, other than 1. We must show that each occurs as a root of the polynomial $h_{\lambda}\left(1, q, \ldots, q^{p-1}\right)$. A $p$ th root of unity will be a primitive $d$ th root of unity for some $d>1$ dividing $p$, and such a root occurs with multiplicity 1 or 0 as a root of $[k]_{q}$, depending upon whether $d$ divides $k$ or not.

By counting multiplicities in the numerator and denominator of (33), it can be seen that for $d$ dividing $p$, each primitive $d$ th root of unity occurs as a root of $h_{r}\left(1, q, \ldots, q^{p-1}\right)$ with multiplicity 1 if $d \chi r$, and 0 if $d \mid r$.

Now suppose $p$ is relatively prime to $n$, and consider $h_{\lambda}\left(1, q, \ldots, q^{p-1}\right)$. If $d \mid p, d>1$, then $d \backslash n$, and hence $d \chi \lambda_{i}$ for some part $\lambda_{i}$ of $\lambda$, since $\sum_{i} \lambda_{i}=n$. The corresponding factor $h_{\lambda_{i}}\left(1, q, \ldots, q^{p-1}\right)$ will then have the primitive $d$ th roots of unity as roots. Hence $1+q+\cdots+q^{p-1}$ divides $h_{\lambda}\left(1, q, \ldots, q^{p-1}\right)$, proving $(3) \Rightarrow(1)$.

The same considerations show that $1+q+\cdots q^{p-1}$ divides $h_{n}\left(1, q, \ldots, q^{p-1}\right)$ if and only if for every $d \mid p, d>1$, we have $d \not n$. This is the same as saying $p$ and $n$ are relatively prime, so $(2) \Rightarrow(3)$.

It remains to show that the polynomials (31) have nonnegative coefficients. There is an algebraic interpretation which implies this, as follows. As in Section 1.5, the initials h.s.o.p. below stand for homogeneous system of parameters.

Proposition 2.5.2. Suppose that $\mathbb{Q}[X]=\mathbb{Q}\left[x_{1}, \ldots, x_{n}\right]$ contains an h.s.o.p. $\theta_{1}, \theta_{2}, \ldots, \theta_{n}$ with the following properties:

(1) $\theta_{1}$ has degree 1 and $\theta_{2}, \ldots, \theta_{n}$ have degree $p$.

(2) $\theta_{1}$ is $S_{n}$ invariant, and $\theta_{2}, \ldots, \theta_{n}$ span a subspace affording the irreducible reflection representation of $S_{n}$. 
Then the Frobenius series of $\mathbb{Q}[X] /\left(\theta_{1}, \ldots, \theta_{n}\right)$ is given by

$$
\mathcal{F}(q)=\sum_{|\lambda|=n} \frac{s_{\lambda}\left(1, q, \ldots, q^{p-1}\right)}{1+q+\cdots q^{p-1}} s_{\lambda}
$$

Proof. This will be a proof sketch, omitting verification of many details.

The idea is to consider the Koszul resolution of $\mathbb{Q}[X] /\left(\theta_{1}, \ldots, \theta_{n}\right)$, which is an exact sequence

$$
0 \rightarrow F_{n} \rightarrow \cdots \rightarrow F_{1} \rightarrow F_{0} \rightarrow \mathbb{Q}[X] /\left(\theta_{1}, \ldots, \theta_{n}\right) \rightarrow 0 .
$$

In this resolution, $F_{k}$ is a free $\mathbb{Q}[X]$ module with basis indexed by $k$-subsets of $\left\{\theta_{1}, \ldots, \theta_{n}\right\}$. Each $F_{k}$ is graded by assigning to each basis element a degree based on the degrees of the $\theta_{i}$. There is an action of $S_{n}$ on everything, and the maps (which I will not describe) are homogeneous of degree 0 and $S_{n}$ equivariant.

It follows from the exactness of (35) that the Frobenius series $\mathcal{F}(q)$ for $\mathbb{Q}[X] /\left(\theta_{1}, \ldots, \theta_{n}\right)$ is given by

$$
\mathcal{F}(q)=\sum_{i=0}^{n}(-1)^{k} \mathcal{F}_{F_{i}}(q) .
$$

Furthermore, the sum on the right is the internal product (the symmetric function operation corresponding to tensor product of $S_{n}$ modules) of the Frobenius series (8) for $\mathbb{Q}[X]$ with the sum

$$
\sum_{i=0}^{n}(-1)^{k} \mathcal{F}_{B_{i}}(q)
$$

where $B_{i}$ is the $\mathbb{Q}$-linear span of the free module basis for $F_{i}$, which is a graded $S_{n}$ module. As it happens, the sum (37) works out to

$$
\frac{1-q}{1-q^{p}} \sum_{|\lambda|=n} \frac{p_{\lambda}}{z \lambda} \prod_{i}\left(1-q^{p \lambda_{i}}\right)
$$

and the internal product of (8) with (38) is exactly (34).

By Proposition 2.5.1 it is necessary that $p$ be relatively prime to $n$ in order for an h.s.o.p. satisfying the hypotheses of Proposition 2.5.2 to exist. We now prove that this condition is also sufficient.

Proposition 2.5.3. An h.s.o.p. satisfying the hypotheses of Proposition 2.5.2 exists whenever $p$ is relatively prime to $n$.

Proof. There is no real choice as to $\theta_{1}$ - up to a scalar multiple, it must be $x_{1}+\cdots+x_{n}$. 
Homogeneous polynomials $\theta_{1}, \ldots, \theta_{n} \in \mathbb{Q}[X]$ form a system of parameters just in case the only solution of the equations $\theta_{1}(X)=0, \ldots, \theta_{n}(X)=0$ is $x_{1}=x_{2}=\cdots=x_{n}=0$.

When $p$ is prime and doesn't divide $n$, the choice $\theta_{i}=x_{i-1}^{p}-x_{i}^{p}$ for $2 \leq i \leq n$ works. In this case the equations $\theta_{i}(X)=0$ say that all $x_{i}^{p}$ are equal and $x_{1}+\cdots+x_{n}=0$. Suppose there is a nonzero solution. Rescaling, we can assume $x_{1}=1$ and hence each $x_{i}$ is a $p$ th root of 1 . Let $\xi$ be a primitive $p$ th root of 1 . Writing $x_{i}$ as $\xi^{a_{i}}$ we have

$$
f(\xi)=\sum_{i} \xi^{a_{i}}=0
$$

Then $f(x)$ is a polynomial with integer coefficients, $f(1)=n$, and $f(x)$ is divisible by the minimal polynomial of $\xi$, namely $1+x+\cdots+x^{p-1}$. Setting $x=1$, this forces $p$ to divide $n$, contrary to assumption.

To complete the proof, we show that if there exist suitable systems of parameters of degrees $p$ and $q$, then there is one of degree $p q$. For this purpose it is best to work modulo $\theta_{1}$, which we always take to be $x_{1}+\cdots+x_{n}$.

Putting $z_{i}=x_{i}-x_{i-1}$ our problem concerns h.s.o.p.s $\left\{\theta_{2}, \ldots, \theta_{n}\right\}$ in $\mathbb{Q}[Z]$ which span a copy of the irreducible reflection representation of $S_{n}$ (as do the $z_{i}$ 's). Up to a linear transformation among the parameters, we can assume that the $\theta_{i}$ permute exactly as the $z_{i}$ do, so that the ring homomorphism $g_{\theta}$ mapping $z_{i}$ to $\theta_{i}$ is $S_{n}$ equivariant. Thus $g_{\theta}$ defines an equivariant polynomial map from the irreducible reflection representation $V$ of $S_{n}$ to itself, and the condition that the $\theta_{i}$ form a system of parameters says precisely that the preimage of 0 under this map is $\{0\}$.

But now, given h.s.o.p.s $\left\{\theta_{2}, \ldots, \theta_{n}\right\}$ and $\left\{\theta_{2}^{\prime}, \ldots, \theta_{n}^{\prime}\right\}$ of degrees $p$ and $q$, the composite map $g_{\theta} \circ g_{\theta^{\prime}}$ again defines an equivariant polynomial map from $V$ to $V$, homogeneous of degree $p q$, and such that the preimage of 0 is $\{0\}$. Hence $g_{\theta} \circ g_{\theta^{\prime}}$ carries the $z_{i}$ onto a suitable h.s.o.p. of degree $p q$.

Notes. Proposition 2.5.3 was a conjecture in an earlier draft of this paper. H. Kraft supplied a crucial part of the proof.

\subsection{The parking function module}

In this section we give a Frobenius series version of Conjecture 2.3.1. There is no reasonable $S_{n}$ action on trees preserving the inversion number or external activity, but there is a related combinatorial structure which serves the purpose, as we now explain.

Definition. A function $f:\{1, \ldots, n\} \rightarrow\{1, \ldots, n\}$ is a parking function if for all $1 \leq k \leq n$, the cardinality of $f^{-1}(\{1, \ldots, k\})$ is at least $k$. 
To motivate this definition, imagine a one-way street with $n$ parking spaces labeled 1 through $n$. There are $n$ cars which want to park along the street, and each car $i$ has a preferred parking space $f(i)$. The cars arrive in succession at the head of the street and each drives immediately to its preferred parking space. If the space is unoccupied, the car parks there; otherwise it continues to the next unoccupied space. If any car reaches the end of the street without having parked, the parking process fails.

It is obvious that a necessary condition for the parking process to succeed is that the preference function $f$ be a parking function, and it is easy to prove that the condition is also sufficient.

Note that the property of being a parking function is invariant under permutation of the cars, that is, under replacement of $f$ by $f \circ \sigma$ for $\sigma \in S_{n}$. In this way $S_{n}$ acts by permutations on the set of parking functions.

PROPOSITION 2.6.1. The number of parking functions for a given $n$ is $(n+1)^{n-1}$, and the permutation action of $S_{n}$ on them is isomorphic to the action on $\mathbb{Z}_{n+1}^{n} / \mathbb{Z}_{n+1}$.

Proof. Consider the parking process on a circular one-way street with parking spaces $1, \ldots, n+1$. Since the street is circular, the process can't fail, and exactly one parking place will be left unoccupied. Among the $n+1$ distinct rotations of any given preference functions $f:\{1, \ldots, n\} \rightarrow\{1, \ldots, n+1\}$, precisely one is a parking function - the one that puts the final unoccupied space at $n+1$.

By making this informal argument precise (which can be done without too much trouble) we can conclude that the permutation action of $S_{n}$ on the set of all functions $f:\{1, \ldots, n\} \rightarrow\{1, \ldots, n+1\}$ is isomorphic to $n+1$ disjoint copies of the action on parking functions, which is equivalent to what what we wanted to show.

The beauty of parking functions is that they have weights, with the same distribution as the inversion statistic for trees.

Definition. The weight $w(f)$ of a parking function $f$ is $n(n+1) / 2-\sum_{i} f(i)$.

The weight is the total distance driven by all cars while searching for a parking place, not counting the initial drive to the preferred spot. Note that it is zero if and only if $f$ is a permutation, and its maximum value $\left(\begin{array}{l}n \\ 2\end{array}\right)$ occurs uniquely for the constant function $f=1$.

PROPOSITION 2.6.2. The sum

$$
\sum_{f} q^{w(f)}
$$

taken over all parking functions for a given $n$ is equal to the inversion enumerator for trees, (21). 
A bijective proof of this proposition is given in [16]. We will give another derivation later using Proposition 4.4.1.

We now have enough machinery to state the Frobenius series version of Conjecture 2.3.1, at least at the representation level. After that, we will work out what it says at the symmetric function level.

Conjecture 2.6.1. Let $V_{n}$ be the following graded $S_{n}$ module. Take the parking functions $f:\{1, \ldots, n\} \rightarrow\{1, \ldots, n\}$ as a $\mathbb{Q}$-basis and grade according to weight. Then $R_{n}$, graded by degree in $X$ (or $Y$ ), is isomorphic as a graded $S_{n}$ module to $\varepsilon \otimes V_{n}$.

Implicit in this conjecture is a value for $\mathcal{F}_{n}(1, q)$ which we now want to make explicit.

The $S_{n}$ orbit of a parking function $f$ determines and is determined by the partition $\mu$ whose parts are the numbers $f(1)-1, \ldots, f(n)-1$, sorted into nonincreasing order. The defining condition for $f$ to be a parking function amounts to the requirement that $\mu \subseteq \delta_{n}$, where $\delta_{n}$ is the partition $(n-1, n-$ $2, \ldots, 1)$ of $\left(\begin{array}{l}n \\ 2\end{array}\right)$, and the expression $\mu \subseteq \delta_{n}$ means the diagram of $\mu$ is contained in that of $\delta_{n}$, which is merely to say that $\mu_{i} \leq n-i$, for each $i$.

The weight of $f$ translates into the quantity $\left(\begin{array}{l}n \\ 2\end{array}\right)-|\mu|$. The symmetric function describing the representation on the orbit corresponding to $\mu$ is $h_{m_{0}(\mu)} h_{m_{1}(\mu)} \cdots$ $h_{m_{n-1}(\mu)}$, provided we agree to assign $\mu$ enough zero parts to give it $n$ parts altogether. Applying $\omega$ for the sign twist, we arrive at the following equivalent formulation of Conjecture 2.6.1.

Conjecture 2.6.2

$$
\mathcal{F}_{n}(1, q)=\sum_{\mu \subseteq \delta_{n}} q^{\left(\frac{n}{2}\right)-|\mu|} e_{\lambda(\mu)},
$$

where $\lambda(\mu)$ is the partition of $n$ whose parts are the multiplicities $m_{i}(\mu)$, and $m_{0}(\mu)$ is defined so that $\sum_{i} m_{i}(\mu)=n$.

In light of Proposition 2.6.1, the expression (41) must specialize to (25) upon setting $q=1$. In other words, the number of $\mu \subseteq \delta_{n}$ with a given $\lambda(\mu)=\lambda$ is equal to $\left({ }_{m_{0}(\lambda), \ldots, m_{n}(\lambda)}^{n+1}\right) /(n+1)$, where $m_{0}(\lambda)$ is defined to make $\sum_{i} m_{i}(\lambda)=n+1$. We leave it as an exercise for the reader to find a direct proof of this fact.

Notes. Gessel informed me about parking functions and was the first to suggest they should have some relationship to the module $R_{n}$. Parking functions were first studied by Konheim and Weiss [14].

\section{7. $t, q$-Catalan numbers}


This section contains no new conjectures, just some interesting cases of those made above. The sign representation appears to play a special role in $R_{n}$, as we shall see more fully in Section 5. Already, we recognize that the conjectures require the multiplicity of the sign character $\varepsilon$ to be the number of $S_{n}$ orbits among parking functions or in $\mathbb{Z}_{n+1}^{n} / \mathbb{Z}_{n+1}$.

This number is the Catalan number

$$
C_{n}=\frac{1}{n+1}\left(\begin{array}{c}
2 n \\
n
\end{array}\right)=\frac{(n+2) \cdots(2 n)}{2 \cdots n}
$$

as can be seen in several ways. For instance, we may appeal to (29), where the coefficient of $s_{\left(1^{n}\right)}$ is $s_{(n)}(1,1, \ldots, 1) /(n+1)$, with $n+1$ ones in the argument, which is equal to (42). Even more directly, we can observe that orbits in $\mathbb{Z}_{n+1}^{n}$ correspond to multisubsets of size $n$ from $\{0, \ldots, n\}$, and there are $\left(\begin{array}{c}2 n \\ n\end{array}\right)$ of these. An alternate approach is to use (41), which has one term per orbit. It is well known that partitions $\mu \subseteq \delta_{n}$ are counted by the Catalan number $C_{n}$. The outlines of the diagrams of $\mu$ can be interpreted as Dyck paths: paths made up of unit upward and rightward steps in the plane, starting at $(0,0)$ and ending at $(n, n)$, which do not fall below the diagonal.

If the conjectures hold, then the quantity

$$
C_{n}(t, q)=\left\langle s_{\left(1^{n}\right)}, \mathcal{F}_{n}(t, q)\right\rangle
$$

must be a $t, q$-analog of the Catalan number $C_{n}$. It would be very interesting to have a plausible combinatorial conjecture as to its value.

Here is a table of the values through $n=5$ :

\begin{tabular}{c|c}
$n$ & $C_{n}(t, q)$ \\
\hline 1 & 1 \\
\hline 2 & $q+t$ \\
\hline 3 & $q^{3}+q t+q^{2} t+q t^{2}+t^{3}$ \\
\hline \multirow{2}{*}{4} & $q^{6}+q^{3} t+q^{4} t+q^{5} t+q^{2} t^{2}+q^{3} t^{2}+q^{4} t^{2}+$ \\
& $q t^{3}+q^{2} t^{3}+q^{3} t^{3}+q t^{4}+q^{2} t^{4}+q t^{5}+t^{6}$ \\
\hline & $q^{10}+q^{6} t+q^{7} t+q^{8} t+q^{9} t+q^{4} t^{2}+q^{5} t^{2}+2 q^{6} t^{2}+$ \\
& $q^{7} t^{2}+q^{8} t^{2}+q^{3} t^{3}+2 q^{4} t^{3}+2 q^{5} t^{3}+q^{6} t^{3}+$ \\
5 & $q^{7} t^{3}+q^{2} t^{4}+2 q^{3} t^{4}+2 q^{4} t^{4}+q^{5} t^{4}+q^{6} t^{4}+$ \\
& $q^{2} t^{5}+2 q^{3} t^{5}+q^{4} t^{5}+q^{5} t^{5}+q t^{6}+2 q^{2} t^{6}+q^{3} t^{6}+$ \\
& $q^{4} t^{6}+q t^{7}+q^{2} t^{7}+q^{3} t^{7}+q t^{8}+q^{2} t^{8}+q t^{9}+t^{10}$
\end{tabular}


Conjectures 2.5.1 and 2.6.2 lead to one-parameter specializations which must hold for $C_{n}(t, q)$. As it turns out, both of these are classical $q$-analogs of $C_{n}$ which are well studied in the literature, so we will take a moment to describe them. There is even in the literature [5] a three-parameter Catalan number $C_{n}(x, a, b)$ which possesses both of the required one-parameter specializations. Unfortunately, this $C_{n}(x, a, b)$ apparently has no two-parameter specialization which agrees with the one-parameter specializations and also with the identity $C_{n}(t, q)=C_{n}(q, t)$, which must certainly hold for (43).

Conjecture 2.5.1 leads to the specialization

$$
q^{\left(\frac{n}{2}\right)} C_{n}\left(q^{-1}, q\right)=\frac{1}{[n+1]_{q}}\left[\begin{array}{c}
2 n \\
n
\end{array}\right]_{q}=\frac{[n+2]_{q} \cdots[2 n]_{q}}{[2]_{q} \cdots[n]_{q}}
$$

The right-hand side is known to be a polynomial with nonnegative coefficients. It counts Catalan words by major index. A Catalan word is a sequence $w_{1} \cdots w_{2 n}$ of $n$ zeroes and $n$ ones, in which the number of ones in an initial segment is not allowed to exceed the number of zeroes. The major index is the sum of those $i$ for which $w_{i}=1$ and $w_{i+1}=0$.

Conjecture 2.6.2 leads to

$$
C_{n}(1, q)=C_{n}(q)
$$

where $C_{n}(q)$ are the $q$-Catalan numbers of Carlitz and Riordan [3]. These can be defined by the recurrence

$$
C_{n+1}(q)=\sum_{i=0}^{n} q^{i} C_{i}(q) C_{n-i}(q)
$$

and count Catalan words by the statistic $\left(\begin{array}{l}n \\ 2\end{array}\right)$ minus the number of inversions, i.e., those $i<j$ for which $w_{i}=1$ and $w_{j}=0$. From this follows (46), since for $\mu \subseteq \delta_{n}$, $|\mu|$ is the number of inversions of a naturally corresponding Catalan word.

Notes. Stanley first noticed that the multiplicity of the sign character is $C_{n}$. Information about the known one-parameter $q$-Catalan numbers and some multiparameter generalizations can be found in [5].

\subsection{Conjectures for $\mathbb{Q}[X, Y, Z]$; problems for $\geq 4$ sets of variables}

There is some computational data on the analog of $R_{n}$ in three and four sets of variables $X, Y, Z, \ldots$ Since I don't necessarily think there is enough evidence to justify the word conjecture, I'll state it as follows.

Fact 2.8.1. For $n \leq 5$ the dimension of $R_{n}(X, Y, Z)$ as a vector space is $2^{n}(n+1)^{n-2}$. The multiplicity of the sign character is 


$$
\frac{(3 n+3) \cdots(4 n+1)}{3 \cdots(n+1)}
$$

The situation for four sets of variables begins to be more problematic. For what it's worth, here are the dimensions for $n \leq 4$.

\begin{tabular}{c|c}
$n$ & $\operatorname{dim} R_{n}(W, X, Y, Z)$ \\
\hline 1 & 1 \\
2 & 5 \\
3 & 55 \\
4 & 996.
\end{tabular}

Large prime factors make a discouraging appearance, e.g., $996=2^{2} \cdot 3 \cdot 83$.

\section{Some theorems}

In this section we prove three theorems about $R_{n}$. None is extremely deep, but they do give some information about the Hilbert and Frobenius series.

\section{1. $\mathfrak{s l}_{2}$ unimodality}

There is an action of $G L_{2}$ on $\mathbb{Q}[X, Y]$ that commutes with the action of $S_{n}$. This can be understood by thinking of the variables $X$ and $Y$ as the rows of a $2 \times n$ matrix $\mathbf{X}$. Then $S_{n}$ permutes the columns, in effect multiplying $\mathbf{X}$ on the right by a permutation matrix. At the same time, we may multiply $X$ on the left by any $2 \times 2$ matrix, getting the $G L_{2}$ action. The $G L_{2}$ action mixes $X$ and $Y$ but does leave invariant the homogeneous components $\bigoplus_{i+j=d}(Q[X, Y])_{i, j}$ of each total degree $d$.

Since $G L_{2}$ commutes with $S_{n}$ it leaves the ideal $I$ invariant, so there is an induced action on $R_{n}$. As we shall see in Section 5, $G L_{2}$ also leaves invariant the space of diagonal harmonics, so we have corresponding actions on $H_{n}$ and $R_{n}$.

To analyze $H_{n}$ as a $G L_{2}$ module we look at the infinitesimal action of the Lie algebra $\mathfrak{s l}_{2}$. Recall that $\mathfrak{s l}_{2}$ has a basis $\{E, F, H\}$ such that $[E, F]=H,[H, E]=$ $2 E$, and $[F, H]=2 F$. In our situation, these are given explicitly as derivations

$$
\begin{aligned}
& E=\sum_{i=1}^{n} y_{i} \partial x_{i} \\
& F=\sum_{i=1}^{n} x_{i} \partial y_{i}
\end{aligned}
$$




$$
H=\sum_{i=1}^{n} y_{i} \partial y_{i}-x_{i} \partial x_{i} .
$$

A weight space is an eigenspace of the operator $H$; its eigenvalue is called the weight. For $H$ given by (49), it is easy to see that the bihomogeneous components of $H_{n}$ are weight spaces, with the weight of $\left(H_{n}\right)_{i, j}$ given by $j-i$.

If $V$ is a finite-dimensional $\mathfrak{o l}_{2}$ module, we write down a Laurent polynomial called the formal character of $V$,

$$
\operatorname{ch}_{V}(q)=\sum_{k} m_{k}(V) q^{k}
$$

where $m_{k}(V)$ is the dimension of the weight space with weight $k$. The formal character contains full information about $V$ as a sum of irreducible $\mathfrak{g l}_{2}$ modules, since there is one such module $V_{m}$ for each integer $m \geq 0$, with character

$$
\operatorname{ch}_{V_{m}}(q)=q^{-m}+q^{-m+2}+\cdots+q^{m-2}+q^{m} .
$$

Because the $S L_{2}$ and $S_{n}$ actions commute, $H_{n}$ decomposes as a direct sum of irreducible $S L_{2} \times S_{n}$ modules, which take the form $V_{m} \otimes W_{\lambda}$, where $V_{m}$ is an irreducible $\mathfrak{s l}_{2}$ module, as just described, and $W_{\lambda}$ is an irreducible $S_{n}$ module. This has the following implications for the Hilbert and Frobenius series.

Proposition 3.1.1. The Frobenius series specialization

$$
\mathcal{F}_{n}\left(q^{-1}, q\right)
$$

is a sum of irreducible $S L_{2} \times S_{n}$ characters of the form

$$
\left(q^{-m}+q^{-m+2}+\cdots+q^{m-2}+q^{m}\right) s_{\lambda} \text {. }
$$

The same is true separately in each total degree. In particular, if we extract those terms from $\mathcal{F}_{n}(t, q)$ of a given total degree $d$ in $t, q$, then set $t=q^{-1}$, the coefficient of each $s_{\lambda}$ in the result is a Laurent polynomial with symmetric and unimodal coefficient sequence. A similar statement holds for the Hilbert series $\mathcal{H}_{n}(t, q)$; in other words, the diagonals corresponding to constant total degree in the diagram (16) are symmetric and unimodal.

Observe, by way of example, that these diagonals in (16) for $n=3$ are 1 , coming from the trivial character, 22 , where each 2 comes from the two-dimensional character of $S_{3}$ (corresponding to the irreducible reflection representation), 232 , consisting of a two-dimensional character in each spot plus the sign character in the middle, and 1111 , coming from the sign character in each spot.

It should be noted that Proposition 3.1.1 does not say that $\mathcal{H}_{n}\left(q^{-1}, q\right)$ has unimodal coefficient sequence, only that the odd degree and even degree parts of it separately have unimodal coefficients. Thus the value for $\mathcal{H}_{n}\left(q^{-1}, q\right)$ 
predicted by Conjecture 2.2.1, which does have unimodal coefficients, remains completely unexplained.

\subsection{Top degree $\left(\begin{array}{l}n \\ 2\end{array}\right)$}

It follows from the observations made in the previous section that in total degree $\left(\begin{array}{l}n \\ 2\end{array}\right)$ we have, at a minimum, one copy of the sign character in every bidegree. The tables suggest that there is nothing more in degree $\left(\begin{array}{l}n \\ 2\end{array}\right)$, and that there is nothing at all in higher degrees. In this section we will prove these two facts.

Our technique is to find "straightening" relations which enable us to reduce everything modulo $I$ to certain monomials, and then to show that in each bidegree of total degree $\left(\begin{array}{c}n \\ 2\end{array}\right)$ these monomials are all congruent to each other. The following lemma provides us with the relations we require.

LEMMA 3.2.1. The polynomials

$$
\left.h_{k}\left(\alpha x_{k}+\beta y_{k}, \ldots, \alpha x_{n}+\beta y_{n}\right)\right|_{\alpha^{r} \beta^{\circ}}
$$

$(1 \leq k \leq n, r+s=k)$ belong to $I$, where $h_{k}$ is the complete homogeneous symmetric function of degree $k$, and the notation $\left.\right|_{\alpha^{r} \beta^{*}}$ means to extract the coefficient of $\alpha^{r} \beta^{s}$.

Proof. Given any homogeneous polynomial $p(X)$, the polynomials $p(\alpha X+$ $\beta Y)\left.\right|_{\alpha^{r} \beta^{s}}$, called polarizations of $p$, can be gotten by repeatedly applying the operator $E=\sum_{i} y_{i} \partial x_{i}$ of (49). Therefore if $p(X)$ belongs to $I$, its polarizations do also, and it suffices to prove $h_{k}\left(x_{k}, \ldots, x_{n}\right) \in I$.

Consider the equation

$$
\prod_{i=1}^{n}\left(1+t x_{i}\right)=\sum_{j \geq 0} t^{j} e_{j}(X) .
$$

Modulo $I$, the right-hand side is 1 , so we have

$$
\prod_{i=1}^{k-1}\left(1+t x_{i}\right) \equiv \prod_{i=k}^{n} \frac{1}{1+t x_{i}}=\sum_{j \geq 0}(-t)^{j} h_{j}\left(x_{k}, \ldots, x_{n}\right) \text {. }
$$

Since the coefficient of $t^{k}$ is zero on the left-hand side, it is zero $(\bmod I)$ on the right, proving the result.

To use the polynomials (54) as straightening relations, we impose a lexicographic order on all monomials in $X, Y$. In this order, we first compare two monomials by their total degree in $x_{1}, y_{1}$, then $x_{2}, y_{2}$, and so forth. For our purposes it doesn't matter how we choose to break ties when all total degrees agree.

In this ordering, the leading, or lexicographically largest, term of (54) is $x_{k}^{r} y_{k}^{a}$. We can use (54) to replace any monomial containing $x_{k}^{r} y_{k}^{s}$ by a combination of lexicographically lesser monomials, modulo $I$. This proves the following. 
COROLLARY 3.2.1. The set of all monomials $m(X, Y)$ with the property that $m(X, Y)$ has total degree less than $k$ in $x_{k}, y_{k}$ for each $k$ spans $R_{n}$. In particular, the homogeneous components $\left(R_{n}\right)_{i, j}$ with $i+j>\left(\begin{array}{l}n \\ 2\end{array}\right)$ are all zero.

The corollary also yields an upper bound on $\mathcal{H}_{n}(1,1)$ of $n !(n+1) ! / 2^{n}$, which unfortunately is a good deal larger than $(n+1)^{n-1}$.

We can say a little more about monomials which vanish modulo $I$.

COROLLARY 3.2.2. If $m(X, Y)$ is a monomial whose total degree in $x_{k}, y_{k}, \ldots, x_{n}, y_{n}$ exceeds $(k-1)+\cdots+(n-1)$ then $m(X, Y) \equiv 0(\bmod I)$.

Proof. Such a monomial is always subject to straightening, and all monomials resulting after a straightening step have the same property. Hence we will eventually reduce the monomial to zero.

By a more delicate application of straightening, we obtain the following theorem.

PROPOSITION 3.2.1. For $i+j=\left(\begin{array}{c}n \\ 2\end{array}\right),\left(R_{n}\right)_{i, j}$ is one-dimensional.

Proof. What we shall do is prove that all the monomials of bidegree $(i, j)$ admitted by Corollary 3.2.1 are actually congruent to each other modulo $I$. Since $i+j=\left(\begin{array}{l}n \\ 2\end{array}\right)$, the admissible monomials have total degree exactly $k-1$ in $x_{k}, y_{k}$ for each $k$. That is, they have the form

$$
x_{1}^{0} y_{1}^{0} x_{1}^{r_{1}} y_{1}^{s_{1}} \cdots x_{n}^{\tau_{n}} y_{n}^{s_{n}}
$$

where $r_{k}+s_{k}=k-1, \sum_{k} r_{k}=i$, and $\sum_{k} s_{k}=j$.

Our first step is to apply a transposition $\sigma=(k, k+1)$ to a monomial of this form and use straightening to express the result. For convenience, let us isolate the $k$ th and $(k+1)$-st variables by writing our monomial as

$$
m(X, Y)=x_{k}^{r} y_{k}^{s} x_{k+1}^{t} y_{k+1}^{u} P
$$

Applying $\sigma$ we get

$$
\sigma m(X, Y)=x_{k}^{t} y_{k}^{u} x_{k+1}^{r} y_{k+1}^{s} P .
$$

Now $x_{k}^{t} y_{k}^{u}$ is the leading term of the straightening relation

$$
\left.h_{k}\left(\alpha x_{k}+\beta_{y k}, \ldots, \alpha x_{n}+\beta y_{n}\right)\right|_{\alpha^{2} \beta^{u}} .
$$

Of the remaining terms in (60), all those involving $x_{l}$ or $y_{l}$ for $l>k+1$ as well as all those of degree exceeding 1 in $x_{k+1}, y_{k+1}$ kill the factor $x_{k+1}^{r} y_{k+1}^{s} P$ in $\sigma m(X, Y)$, modulo $I$. This is a consequence of Corollary 3.2.2. As a result, 
if we multiply the 'tail' $x_{k+1}^{r} y_{k+1}^{s} P$ by (60), we obtain the following congruence modulo $I$ :

$$
\begin{aligned}
\left(\begin{array}{c}
k \\
t
\end{array}\right) \sigma m(X, Y) \equiv & -\left(\left(\begin{array}{c}
k-1 \\
t-1
\end{array}\right) x_{k}^{t-1} y_{k}^{u} x_{k+1}^{r+1} y_{k+1}^{s}\right. \\
& \left.+\left(\begin{array}{c}
k-1 \\
t
\end{array}\right) x_{k}^{t} y_{k}^{u-1} x_{k+1}^{r} y_{k+1}^{s+1}\right) P
\end{aligned}
$$

or

$$
k \sigma m(X, Y) \equiv-\left(t x_{k}^{t-1} y_{k}^{u} x_{k+1}^{r+1} y_{k+1}^{s}+u x_{k}^{t} y_{k}^{u-1} x_{k+1}^{r} y_{k+1}^{s+1}\right) P .
$$

This even holds for $t=0$ or $u=0$, since the term involving a negative exponent has vanishing coefficient.

Now comes a trick: we'll apply the formula (62) twice to get an expression for $\sigma^{2} m(X, Y)=m(X, Y)$. The result is

$$
\begin{aligned}
k^{2} x_{k}^{r} y_{k}^{s} x_{k+1}^{t} y_{k+1}^{u} P \equiv & \left(r u x_{k}^{r-1} y_{k}^{s+1} x_{k+1}^{t+1} y_{k+1}^{u-1}+((r+1) t\right. \\
& \left.+(s+1) u) x_{k}^{r} y_{k}^{s} x_{k+1}^{t} y_{k+1}^{u}+s t x_{k}^{r+1} y_{k}^{s-1} x_{k+1}^{t-1} y_{k+1}^{u+1}\right) P
\end{aligned}
$$

Notice that each monomial in this expression has the form $x_{k}^{a} y_{k}^{b} x_{k+1}^{c} y_{k+1}^{d} P$, where $a+b=k-1, c+d=k, a+c=f$, say, and $b+d=g$, where $f=r+t$ and $g=s+u$ are the same for all the monomials. Let us fix $f$ and $g$ such that $f+g=(k-1)+k$, and also fix $P$, so that all the rest is determined by $a$, that is, we restrict our attention to monomials of the form

$$
m_{a}(X, Y)=x_{k}^{a} y_{k}^{k-1-a} x_{k+1}^{f-a} y_{k+1}^{k-f+a} P=x_{k}^{a} y_{k}^{k-1-a} x_{k+1}^{f-a} y_{k+1}^{g-k+1+a} P
$$

for the range $a_{0}=\max (0, f-k) \leq a \leq \min (k-1, f)=a_{1}$ (note this range is nonempty since $0 \leq f \leq(k-1)+k)$.

In this notation, the equation (63) becomes

$$
\begin{aligned}
m_{a} \equiv & \frac{a(k-f+a)}{k^{2}} m_{a-1}+\frac{(a+1)(f-a)+(k-a)(k-f+a)}{k^{2}} m_{a} \\
& +\frac{(k-1-a)(f-a)}{k^{2}} m_{a+1} .
\end{aligned}
$$

This can also be written

$$
\left[m_{a_{0}} \cdots m_{a_{1}}\right](\mathbf{I}-\mathbf{M}) \equiv 0
$$

in matrix notation, where $\mathbf{M}$ is seen to be a square tridiagonal matrix in which all entries on the three diagonals are strictly positive and each column sums to 1. It follows from the elementary theory of Markov processes, applied to the random walk with transition matrix $\mathbf{M}$, that $\mathbf{M}$ has a one-dimensional eigenspace with eigenvalue 1 , and hence that the only solutions of (66) are multiples of $[1 \cdots 1]$. In other words, the monomials $m_{a}$ are all conguent modulo $I$. 
These congruences mean that given a monomial $x_{k}^{r} y_{k}^{s} x_{k+1}^{t} y_{k+1}^{u} P$ as in (58), we are free to redistribute the exponents so as to put all the $x$ 's before all the $y$ 's. That is, we can arrange to have either no $y_{k}$ 's (if $r+t \geq k-1$ ) or no $x_{k+1}$ 's (if $r+t \leq k-1$ ). Applying this repeatedly for various $k$, we reduce to a monomial, still admissible by Corollary 3.2.2, which contains no $x_{k} y_{l}$ with $l<k$. Since there is clearly only one such monomial of bidegree $(i, j)$, the proof is complete.

Notes. The proof of Lemma 3.2.1 is due to Garsia. The proof given here of Proposition 3.2.1 is due jointly to Garsia and myself. A somewhat simpler proof of the part of Corollary 3.2.1 asserting that $\left(R_{n}\right)_{i, j}=0$ for $i+j>\left(\begin{array}{l}n \\ 2\end{array}\right)$ had been found earlier by $\mathrm{N}$. Wallach.

\subsection{Bi-degrees $(-, 1)$}

Conjecture 2.6.2 predicts that the space $\left(H_{n}\right)_{-, 1}$ of diagonal harmonics linear in the $Y$ variables should have dimension $(n-1) n ! / 2$, and that after twisting by the sign character, it should be isomorphic as an $S_{n}$ module to $n-1$ copies of the induced representation $1 \uparrow_{S_{2}}^{S_{n}}$, that is, of the permutation representation on cosets of the subgroup $S_{2}$.

Here I will sketch a proof of this due to J. Alfano and N. Wallach. More detail will be given in a separate publication by them. The result gives a little more: the character of $\left(H_{n}\right)_{d, 1}$ for every $d$.

THEOREM 3.3.1. The Frobenius series

$$
\sum_{d} t^{d} \phi\left(\left(H_{n}\right)_{d, 1}\right)
$$

is equal to

$$
\left(1+t+\cdots+t^{n-2}\right) \sum_{\lambda} t^{\left(n^{-1}\right)} K_{\lambda \mu}\left(t^{-1}\right) s_{\lambda}
$$

where $\mu=\left(2,1^{n-2}\right)$, and $K_{\lambda \mu}(t)$ are the "t-Kostka coefficients" expanding the Schur function $s_{\lambda}$ in terms of Hall-Littlewood polynomials $P_{\mu}(t)$ (cf. [17]).

Note that since $K_{\lambda \mu}(1)$ are the ordinary Kostka coefficients, (68) with $t=1$ reduces to $(n-1) h_{2,1^{n-2}}$, in agreement with Conjecture 2.6.2.

Proof of Theorem 3.3.1. In Section 5 we shall find a family of operators which carry diagonal harmonics into diagonal harmonics; these include

$$
E=\sum_{i=1}^{n} y_{i} \partial x_{i}
$$


from (49) and

$$
D=\sum_{i=1}^{n} y_{i} \partial y_{i} \partial x_{i}
$$

The Vandermonde determinant $\Delta(X)=\prod_{i<j}\left(x_{i}-x_{j}\right)$ is harmonic, so $E \Delta(X), D^{k}$ $E \Delta(X)$ for all $k$, and all their $x$ derivatives belong to $\left(H_{n}\right)_{-, 1}$.

Now $D^{n-2} E \Delta(X)$ is readily computed to be (apart from a constant factor)

$$
\sum_{i=1}^{n}(-1)^{i} y_{i} \Delta\left(x_{1}, \ldots, x_{i-1}, x_{i+1}, \ldots, x_{n}\right),
$$

and it follows from the results of $[2,7]$ that the space of all $x$ derivatives of this alternating polynomial has Frobenius series

$$
\sum_{\lambda} t^{\left(\frac{n-1}{2}\right)} K_{\lambda \mu}\left(t^{-1}\right) s_{\lambda}
$$

Let $A_{k}(0 \leq k \leq n-2)$ be the space of all $x$ derivatives of $D^{k} E \Delta(X)$. The operator $D$ induces an $S_{n}$ equivariant surjection $A_{k} /\left(A_{k} \cap A_{k+1}\right) \rightarrow A_{k+1} /\left(A_{k+1} \cap A_{k+2}\right)$ which lowers $x$ degree by 1 . This implies that the Frobenius series of the space $A=\sum_{k} A_{k} \subseteq\left(H_{n}\right)_{-, 1}$ is coefficientwise at least that given by (68), with equality if $\operatorname{dim} A \leq(n-1) n ! / 2$.

Now consider the space $T$ of all polynomials $\sum_{i} y_{i} f_{i}$, where each $f_{i}$ is a harmonic polynomial in $X$ and $\sum_{i} f_{i}=0$. Obviously $\operatorname{dim} T=(n-1) n$ !, and we have $A \subseteq\left(H_{n}\right)_{-, 1} \subseteq T$.

Let $B$ be the orthogonal complement of $A$ in $T$, with respect to the apolar form. Apart from a constant factor, $D^{k} E \Delta(X)=E_{k+1} \Delta(X)$, where

$$
E_{k}=\sum_{i=1}^{n} y_{i} \partial x_{i}^{k}
$$

Applying the adjoints of the operators $E_{k}$, one shows that $\sum_{i} y_{i} f_{i} \in B$ if and only if

$$
\sum_{i} \partial x_{i}^{k} f_{i}\left(\partial x_{1}, \ldots, \partial x_{n}\right) \Delta(X)=0
$$

Since the $f_{i}$ are harmonic, we may write $f_{i}=g_{i}\left(\partial x_{1}, \ldots, \partial x_{n}\right) \Delta(X)$. The $g_{i}(X)$ may be taken harmonic, and the expression for $f_{i}$ is then unique. Now $\sum_{i} y_{i} f_{i}$ is diagonal harmonic just in case the operators $\sum_{i} \partial y_{i} \partial x_{i}^{k}$ kill it for $k>0$, that is, just in case

$$
\sum_{i} \partial x_{i}^{k} g_{i}\left(\partial x_{1}, \ldots, \partial x_{n}\right) \Delta(X)=0
$$


Comparing (74) and (75) shows that the spaces $B$ and $\left(H_{n}\right)_{-, 1}$ have the same dimension, and this dimension is at least $(n-1) n ! / 2$, since $B+\left(H_{n}\right)_{-, 1}=T$. Equivalently, the dimension of $A$ is at most $(n-1) n ! / 2$, which as we have already seen implies $\operatorname{dim} A=(n-1) n ! / 2$ and hence $\operatorname{dim} B=\operatorname{dim}\left(H_{n}\right)_{-, 1}=(n-1) n ! / 2$. Therefore $\left(H_{n}\right)_{-, 1}=A$ and the Frobenius series of this space is given by (68).

\section{Lagrange inversion}

There is a remarkable relationship between the conjectures for the Frobenius series of $R_{n}$ and certain $q$-analogs of the Lagrange inversion formula, which we discuss in this section.

\subsection{Ordinary Lagrange inversion and 2.4.}

Our first observation is that Conjecture 2.4.2 for the "ungraded" Frobenius series $\mathcal{F}_{n}(1,1)$ reduces in a certain sense to the classical Lagrange inversion formula.

To explain this we must place Lagrange inversion in a symmetric function setting. For this purpose, we think of the ring of symmetric functions abstractly as the polynomial ring

$$
\mathbb{Q}\left[h_{1}, h_{2}, \ldots\right],
$$

where the $h_{k}$ 's, normally understood to be the complete homogeneous symmetric functions, are now understood formally as indeterminates.

From this point of view, we can express a generic formal power series of one variable with zero constant term and unit linear term, in the form

$$
z H(z)=z+h_{1} z^{2}+h_{2} z^{3}+\cdots=\sum_{k \geq 0} h_{k} z^{k+1},
$$

with the usual convention $h_{0}=1$. Relative to (77), the elementary symmetric functions become the coefficients of

$$
z / H(-z)=z+e_{1} z^{2}+e_{2} z^{3}+\cdots=\sum_{k \geq 0} e_{k} z^{k+1} .
$$

The Lagrange inversion formula gives the coefficients of the functional composition inverse of (77). We shall write it in a form giving

$$
w H^{*}(w)=w+h_{1}^{*} w^{2}+h_{2}^{*} w^{3}+\cdots=\sum_{k \geq 0} h_{k}^{*} w^{k+1}
$$

where $H^{*}(w)$ is defined by the functional inverse relationship

$$
w=z H(-z) \quad \Leftrightarrow \quad z=w H^{*}(w) .
$$


Given (79) and (80), the Lagrange inversion formula is

$$
h_{n}^{*}=\left.\frac{1}{n+1} \frac{1}{H(-z)^{n+1}}\right|_{z^{n}},
$$

where the vertical bar indicates taking a coefficient. Applying (78), this becomes in terms of elementary symmetric functions

$$
h_{n}^{*}=\sum_{|\lambda|=n} \frac{1}{n+1}\left(\begin{array}{c}
n+1 \\
m_{0}(\lambda), m_{1}(\lambda), \ldots, m_{n}(\lambda)
\end{array}\right) e_{\lambda} .
$$

where $\sum_{i} m_{i}(\lambda)=n+1$. This is exactly the value proposed by Conjecture 2.4 .2 for $\mathcal{F}_{n}(1,1)$.

Notes. I. Macdonald was the first to point out that the quantity (82) conjectured for $\mathcal{F}_{n}(1,1)$ is the same as $h_{n}^{*}$.

\section{2. 'Trivial' $q$-Lagrange inversion and $2.2,2.5,2.7$}

Once it is seen that Conjecture 2.4.2 takes the form $\mathcal{F}_{n}(1,1)=h_{n}^{*}$, it is natural to ask whether Conjectures 2.5.1 and 2.6.2 might reflect reasonable $q$-analogs of Lagrange inversion. They do, as we now explain. First we treat Conjecture 2.5.1. Recall that the conjecture asserts

$$
q^{\left(\frac{1}{2}\right)} \mathcal{F}_{n}\left(q^{-1}, q\right)=\sum_{|\lambda|=n} \frac{s_{\lambda^{\prime}}\left(1, q, \ldots, q^{n}\right)}{1+q+\cdots+q^{n}} s_{\lambda}
$$

The Cauchy formula

$$
\sum_{\lambda} s_{\lambda^{\prime}}(X) s_{\lambda}(Y)=\prod_{i, j}\left(1+x_{i} y_{j}\right)
$$

can be written, in the notation of (78), as

$$
\sum_{|\lambda|=n} s_{\lambda^{\prime}}(X) s_{\lambda}=\left.\prod_{i} \frac{1}{H\left(-x_{i} z\right)}\right|_{z^{n}} .
$$

Letting $X=\left\{1, q, \ldots, q^{n}\right\}$, we see that the right-hand side of (83) becomes the following straightforward $q$-analog of the Lagrange inversion formula (81).

$$
q^{\left(\frac{n}{2}\right)} \mathcal{F}_{n}\left(q^{-1}, q\right)=\left.\frac{1}{1+q+\cdots+q^{n}} \frac{1}{H(-z) H(-q z) \cdots H\left(-q^{n} z\right)}\right|_{z^{n}} .
$$

At present, equation (86), however striking, has not proven very useful. It would be nice, for instance, if the $q$-analog relationship between (86) and (81) 
could somehow explain the fact that the coefficient of $s_{\lambda}$ in (83) is a polynomial with nonnegative coefficients.

One pleasant application that can be made of (86) is to show that the Frobenius series Conjecture 2.5.1 does in fact generalize the corresponding Hilbert series Conjecture 2.2.1, as it should.

To convert from Frobenius to Hilbert series, we must replace $e_{\lambda}$ by $\left(\begin{array}{l}n \\ \lambda\end{array}\right)$, which is the same as specializing $e_{k}$ to $1 /(k$ !) for all $k$ and then multiplying by $n$ !. Putting $e_{k}=1 /(k !)$, we have $1 / H(-z)=e^{z}$, so (86) becomes

$$
\begin{aligned}
q^{\left(\frac{n}{2}\right)} \mathcal{H}_{n}\left(q^{-1}, q\right) & =\left.n ! \frac{1}{1+q+\cdots+q^{n}} e^{\left(1+q+\cdots+q^{n}\right) z}\right|_{z^{n}} \\
& =\left(1+q+\cdots+q^{n}\right)^{n-1},
\end{aligned}
$$

in accord with Conjecture 2.2.1.

The Catalan number formula (43) is another interesting specialization of (86). Every $e_{\lambda}$ contains $s_{\left(1^{n}\right)}$ with coefficient 1 , so the specialization we require is $e_{k}=1$ for all $k$, or $1 / H(-z)=1 /(1-z)$. With this, (86) reduces to

$$
q^{\left(\frac{n}{2}\right)} C_{n}\left(q^{-1}, q\right)=\left.\frac{1}{1+q+\cdots+q^{n}} \frac{1}{(1-z)(1-q z) \cdots\left(1-q^{n} z\right)}\right|_{z^{n}} .
$$

This $q$-Lagrange formula for the $q$-Catalan numbers given by (43) is the same as the one considered in [5].

\subsection{A-G-G Lagrange inversion}

When we consider Conjecture 2.6.1, we arrive at a $q$-analog of Lagrange inversion much deeper and more satisfactory than that given by (86). This is the theory of Andrews, Garsia, and Gessel, $[1,6,10]$, which was discovered in different forms by each of these three people, and shown to be all one theory by Garsia. In this section we review the A-G-G theory, essentially in the formulation of [6].

The key is to introduce a $q$-analog of the functional composition inverse relationship (79-80). The operation of $q$-functional composition is defined by

$$
\left(F \circ_{q} G\right)(z)=\sum_{n} f_{n} G(z) G(q z) \cdots G\left(q^{n-1} z\right), \quad \text { where } F(z)=\sum_{n} f_{n} z^{n} .
$$

A fundamental theorem of [6] justifies this as natural by showing that for $F$ and $G$ without constant term, the two inverse relationships

$$
F \circ_{q} G=z \text { and } G \circ_{q^{-1}} F=z
$$

are equivalent to one another and also to

$$
\left(\Phi \circ_{q^{-1}} F\right) \circ_{q} G=\Phi=\left(\Phi \circ_{q} G\right) \circ_{q^{-1}} F \text { for all } \Phi \text {. }
$$


In addition Garsia gives a $q$-Lagrange inversion formula to find either of $F$ or $G$ from the other. This formula will not be stated here; instead a more combinatorially explicit formula will be given in the next section.

What we will do here is use (90) to define a $q$-analog $h_{n}^{*}(q)$ of the $h_{n}^{*}$ defined by $(79-80)$. For this we put

$$
\begin{aligned}
& F(z)=z H(-z)=z-h_{1} z^{2}+h_{2} z^{3}+\cdots \quad=\sum_{k \geq 0}(-1)^{k} h_{k} z^{k+1}, \\
& G(z)=z \tilde{H}^{*}(z ; q)=z+q h_{1}^{*}(q) z^{2}+q^{2} h_{2}^{*}(q) z^{3}+\cdots=\sum_{k \geq 0} q^{k} h_{k}^{*}(q) z^{k+1}
\end{aligned}
$$

and require $F$ and $G$ to be related by (89). Equivalently, $h_{k}^{*}(q)$ is defined by the single equation

$$
z=\sum_{k \geq 0} q^{-\left(\frac{k}{2}\right)} h_{k}^{*}(q) z^{k+1} H(-z) H\left(-q^{-1} z\right) \cdots H\left(-q^{-k} z\right) .
$$

\subsection{Solution of $A-G-G$ inversion problem and $2.3,2.6,2.7$}

It happens that (92-93) can be solved explicitly and that the solution is none other than the value proposed by Conjecture 2.6 .2 for $\mathcal{F}_{n}(1, q)$.

PROPOSITION 4.4.1. The $q$-Lagrange inversion coefficients $h_{n}^{*}(q)$ given implicitly by (92-93) are given explicitly by

$$
h_{n}^{*}(q)=\sum_{\mu \subseteq \delta_{n}} q^{\left(\frac{n}{2}\right)-|\mu|} e_{\lambda(\mu)},
$$

where $1 / H(-z)=\sum_{k \geq 0} e_{k} z^{k}$, as in (78), and $\lambda(\mu)$ is the partition whose parts are the multiplicities $m_{i}(\mu), 0 \leq i \leq n-1, m_{0}$ being defined so that $|\lambda|=n$.

Proof. Divide (93) by $z H(-z)$ and replace $z$ with $q z$, obtaining

$$
\begin{aligned}
1 / H(-q z) & =\sum_{k \geq 0} q^{k} h_{k}^{*}(q) z H(-z) q^{-1} z H\left(-q^{-1} z\right) \cdots q^{1-k} z H\left(-q^{1-k} z\right) \\
& =\mathscr{H}^{*}(z ; q) \circ_{q^{-1}}(z H(-z)) .
\end{aligned}
$$

Applying (91) with $\Phi=\mathscr{H}^{*}(z ; q)$ gives

$$
\tilde{H}^{*}(z ; q)=(1 / H(-q z)) \circ_{q}\left(z \tilde{H}^{*}(z ; q)\right) \text {. }
$$

Comparing coefficients of $z^{n}$ we arrive at the following recurrence for $h_{n}^{*}(q), n>0$ :

$$
h_{n}^{*}(q)=\sum_{k=1}^{n} q^{\left(\frac{k}{2}\right)} e_{k} \sum_{\substack{n_{1}+\ldots+n_{k}=n-k \\ n_{i} \geq 0}} \prod_{i=1}^{k} q^{(i-1) n_{i}} h_{n_{i}}^{*}(q) .
$$


It remains to verify that (94) solves the recurrence (97). We will only say how to associate with each $\mu \subseteq \delta_{n}$ numbers $k$ and $n_{1}, \ldots, n_{k}$ leaving it to the reader to check in detail that those terms in (94) belonging to a given $k$ and $n_{1}, \ldots, n_{k}$, are precisely accounted for by the term in (97) for that $k$ and $n_{1}, \ldots, n_{k}$.

The rules are as follows. First of all, $k$ is the number of 0 parts in $\mu$, that is, $\mu_{n-k}$ is the last positive part of $\mu$, and $k=n$ if $\mu=(0,0, \ldots, 0)$. For each $1 \leq i \leq k$, let $p_{i}$ be the largest index for which $\mu_{p_{i}}$ is positive and $\mu_{p_{i}}>n-i-p_{i}$, or zero if there is no such index. In pictorial terms, the $p_{i}$ th row of $\mu$ is the last one that does not fit inside the diagram of $\delta_{n-i}$.

Obviously $p_{1} \leq p_{2} \leq \cdots \leq p_{k}$, since the condition $\mu_{p_{i}} \geq n-i-p_{i}$ becomes weaker as $i$ increases. Also it is clear that $p_{k}=n-k$. Now define $n_{i}$ by setting

$$
p_{i}=n_{1}+\cdots+n_{i}
$$

for each $i$, so the $n_{i}$ are nonnegative integers and $n_{1}+\cdots+n_{k}=n-k$, as required.

Now it is only necessary to check that as we sum over partitions $\mu$ with fixed $k$ and $n_{1}, \ldots, n_{k}$, the contribution to $q^{\left(\frac{()}{2}\right)-|\mu|} e_{\lambda(\mu)}$ due to parts $\mu_{p}$ with $p_{i-1} \leq p \leq p_{i}$ is correctly accounted for by the factor $q^{(i-1) n_{i}} h_{n_{i}}^{*}(q)$. Since the contribution due to the $k$ zero parts $\mu_{n-k+1}, \ldots, \mu_{n}$ is $q^{\left(\frac{k}{2}\right)} e_{k}$, this completes the proof.

Proposition 4.4.1 may be applied to derive Proposition 2.6.2, using a result of Gessel [10]. Letting

$$
J_{n}(q)=\sum_{T} q^{\operatorname{inv}(T)}
$$

be the inversion enumerator for trees on $\{0,1, \ldots, n\}$, Proposition 2.6 .2 asserts, in effect, that when $e_{k}=1 /(k !)$, we have

$$
n ! h_{n}^{*}(q)=J_{n}(q)
$$

To prove (100), consider the generating function

$$
J(z ; q)=\sum_{n \geq 0} \frac{J_{n}(q) z^{n}}{n !}
$$

Using $q$-Lagrange inversion and the Mallows-Riordan generating function for $J_{n}(q)$, Gessel obtains a formula $[10,(14.3)]$ which in the notation used here becomes

$$
J(z ; q)=e^{z} \circ_{q}(z J(z ; q)) .
$$

In this equation, we may replace $z$ by $q z$ and compare with (96), taking $H(z)=e^{z}$, to see that for the specialization $e_{k}=1 /(k !)$,

$$
J(q z ; q)=\tilde{H}^{*}(z ; q) .
$$


This is the generating function version of (100).

It is also interesting to apply Proposition 4.4.1 using the Catalan number specialization $e_{k}=1, H(z)=(1+z)$. Equation (93) becomes

$$
z=\sum_{k \geq 0} q^{\left({ }^{k}\right)} h_{k}^{*}\left(q^{-1}\right) z^{k+1}(1-z)(1-q z) \cdots\left(1-q^{k} z\right),
$$

and using Proposition 4.4.1 for $h_{k}^{*}$, we obtain

$$
z=\sum_{k \geq 0} q^{\left({ }^{k}\right)} C_{k}\left(q^{-1}\right) z^{k+1}(1-z)(1-q z) \cdots\left(1-q^{k} z\right),
$$

where $C_{k}(q)$ are the Carlitz-Riordan $q$-Catalan numbers from (46-47). It can be shown directly that (105) and (47) have the same solution. This is done in [5], where (105) is taken as the definition of the Carlitz-Riordan $q$-Catalan numbers.

Notes. Proposition 4.4 .1 or something like it has apparently been known to Garsia and possibly other people for some time, but it has not previously appeared in the literature. D. Singer has just introduced in his Ph.D. thesis a far-reaching generalization of A-G-G Lagrange inversion that also includes the novel $q$-Lagrange inversion of Garsia and Remmel [8]. There is a hope that in Singer's framework it may be possible to define a $(t, q)$-Lagrange inversion which could account for the full Frobenius series $\mathcal{F}_{n}(t, q)$.

\subsection{A conjectured exact sequence; abstract existence for $n \leq 6$}

Using Proposition 4.4.1, Conjecture 2.6.2 takes the form

$$
z=\sum_{n \geq 0} q^{\left(\frac{n}{2}\right)} \mathcal{F}_{n}\left(1, q^{-1}\right) z^{n+1} H(-z) H(-q z) \cdots H\left(-q^{n} z\right)
$$

Equating coefficients of $z^{n+1}$ we obtain

$$
\left.\sum_{k=0}^{n}(-1)^{k} F_{n-k}(q) H(z) H(q z) \cdots H\left(q^{n-k} z\right)\right|_{z^{k}}=0,
$$

where $F_{n}(q)=q^{\left(\frac{n}{2}\right)} \mathcal{F}_{n}\left(1, q^{-1}\right)$. Apart from the sign $(-1)^{k}$, each term in the sum is the Frobenius series of a graded $S_{n}$ module. This suggests that (107) might be explained by an appropriate exact sequence of graded $S_{n}$ modules. In this section we describe the modules which ought to form the supposed exact sequence. We do not, however, find any maps. To give suitable maps and prove exactness would, of course, establish Conjecture 2.6.2.

PROPOSITION 4.5.1. Given $n$ and $k \leq n$, consider a graded $S_{n}$ module constructed as follows. Grade the $S_{n-k}$ module $R_{n-k}$ by the complement of degree in $X$, 
so its Frobenius series is $F_{n-k}(q)$. Tensor this with the $S_{k}$ module $\mathbb{Q}\left[x_{1}, \ldots, x_{k}\right] /$ $\left(x_{1}^{n+1-k}, \ldots, x_{k}^{n+1-k}\right)$, graded in the normal way. Finally induce the resulting $S_{k} \times S_{n-k}$ module to $S_{n}$. The module so constructed has Frobenius series

$$
\left.F_{n-k}(q) H(z) H(q z) \cdots H\left(q^{n-k} z\right)\right|_{z^{k}}
$$

Proof. All we need to prove is that the Frobenius series of $\mathbb{Q}\left[x_{1}, \ldots, x_{k}\right] /$ $\left(x_{1}^{n+1-k}, \ldots, x_{k}^{n+1-k}\right)$ is given by

$$
\left.H(z) H(q z) \cdots H\left(q^{n-k} z\right)\right|_{z^{k}}
$$

In this situation, $x_{1}^{n+1-k}, \ldots, x_{k}^{n+1-k}$ is an h.s.o.p., and we can evaluate the Frobenius series in question from the Koszul resolution, as in the proof of Proposition 2.5.2. Omitting details, what results is the internal product of

$$
\left.\prod_{i=0}^{\infty} H\left(q^{i} z\right)\right|_{z^{k}}
$$

with

$$
\left.\frac{H(z)}{H\left(q^{n+1-k} z\right)}\right|_{z^{k}},
$$

and this works out to (109).

COROLLARY 4.5.1. Suppose there exists an exact sequence

$$
0 \rightarrow R_{n}^{(n)} \rightarrow R_{n}^{(n-1)} \rightarrow \cdots \rightarrow R_{n}^{(0)}=R_{n} \rightarrow 0
$$

of graded $S_{n}$ modules, where $R_{n}^{(k)}$ is as described in the preceding proposition. Then Conjecture 2.6.2 holds for $n$.

Given the Frobenius series of the modules $R_{n}^{k}$, one can decide using only numerology whether or not an exact sequence of the form (112) exists. The condition is that

$$
\sum_{k=0}^{m}(-1)^{m-k} \mathcal{F}_{R_{n}^{(k)}}(q)
$$

must have nonnegative coefficients for all $0 \leq m \leq n$. This condition has been verified for $n \leq 6$. The computations even suggest that the coefficient of each $s_{\lambda}$ in (113) is a polynomial in $q$ with unimodal (but not symmetric) coefficients.

Of course, to give a formula defining explicit maps is another matter entirely.

Notes. The idea of interpreting (107) as an exact sequence was first suggested by Stanley. 


\section{Structure of $H_{n}$}

Recall from Section 1.5 that for the ordinary action of a reflection group, the space of harmonics is spanned by the discriminant (for $S_{n}$, the Vandermonde determinant) and its partial derivatives of all orders. In this section we explore to what extent the diagonal harmonics have a similar structure.

Our method is to introduce certain invariant differential operators which transform harmonics into harmonics. By applying these to the discriminant in one set of variables and its derivatives, we generate a large space of harmonics. In the case of $S_{n}$, we conjecture that the space so generated exhausts all the harmonics, a situation which if true would have a number of interesting consequences.

For reflection groups in general, it is not true that all harmonics can be generated in the manner just described. Some discussion of the possible significance of this failure can be found in Section 7.

\subsection{Operators $E_{p}$ and operator conjecture}

Recall that a polynomial is diagonal harmonic if it is killed by the operators

$$
P_{h, k}=\sum_{i=1}^{n} \partial x_{i}^{h} \partial y_{i}^{k} \quad(h+k>0),
$$

or more generally, by any $S_{n}$ invariant differential operator homogeneous of strictly negative total degree $\left(\partial x_{i}\right.$ and $\partial y_{i}$ have degree -1$)$.

For $p>0$, define

$$
E_{p}=\sum_{i=1}^{n} y_{i} \partial x_{i}^{p}
$$

In particular, the $E$ of (49) is $E_{1}$. We have the commutator

$$
\left[E_{p}, P_{h, k}\right]=-k P_{h, k-1},
$$

which immediately implies that $E_{p}$ carries the space $H_{n}$ into itself. The operators $E_{p}$ raise $y$ degree and lower $x$ degree. The unique (up to constant factor) diagonal harmonic of minimal $y$ degree and maximal $x$ degree is the Vandermonde $\Delta(X)$, suggesting the following conjecture.

Conjecture 5.1.1 (operator conjecture). The space $H_{n}$ is the smallest space containing $\Delta(X)$ and closed under the action of the operators $E_{p}$ for all $p$ and $\partial x_{i}$ for all $i$.

Note that the operators in question commute with each other and that $E_{\mathrm{p}}$ kills $\Delta(X)$ for $p>n-1$. Thus the conjecture would mean that the space $H_{n}$ consists 
of all derivatives of harmonics of the form $E_{1}^{e_{1}} \cdots E_{n-1}^{e_{n-1}} \Delta$. These must then be all the alternating harmonic polynomials; by Conjecture 2.4 .1 the number of linearly independent ones should be the Catalan number $C_{n}$.

The operator conjecture 5.1.1 has been verified by computer for $n \leq 6$. The space considered in the conjecture clearly coincides with the harmonics in degrees $(-, 0)$, and the proof of Theorem 3.3.1 shows that these spaces also coincide in degrees $(-, 1)$. Corollary 3.2.1 and Proposition 3.2.1 may also be regarded as evidence supporting the conjecture, since it explains both of them.

Limited computational evidence suggests that analogs of the operator conjecture hold for more than two sets of variables.

\subsection{Full Lie algebra of operators generated by $E_{\mathrm{p}}$ and $\partial X$}

The operators $E_{p}$ belong to a larger Lie algebra spanned by operators of the form

$$
\begin{aligned}
\sum_{i=1}^{n} y_{i} \partial x_{i}^{h} \partial y_{i}^{k} f\left(\partial x_{1}, \ldots, \partial x_{i-1}, \partial x_{i+1}, \ldots, \partial x_{n}, \partial y_{1}, \ldots\right. \\
\\
\left.\partial y_{i-1}, \partial y_{i+1}, \ldots, \partial y_{n}\right)
\end{aligned}
$$

or a similar form with $x$ and $y$ interchanged. Here $f$ is any diagonally $S_{n-1}$ invariant homogeneous polynomial and we require $h+k+\operatorname{deg}(f)>0$. Indeed, (117) represents the general term of an $S_{n}$ invariant polynomial differential operator linear in the $x_{i}$ and $y_{i}$ and homogeneous of nonpositive degree. The commutator of two such operators is another, so they form a Lie algebra. Also, the commutator of any such operator with an $S_{n}$ invariant pure differential operator of negative degree is again an $S_{n}$ invariant pure differential operator of negative degree, which implies that these operators carry the space $H_{n}$ into itself. The $\mathrm{sl}_{2}$ operators $E, F, H$ of (49) and the operator $D$ of $(70)$ are examples of operators of the form (117).

To study Conjecture 5.1.1, we are free to use all operators of the form (117), by the following proposition.

Proposition 5.2.1. The smallest space containing $\Delta(X)$ and closed under the action of all operators of the form (117) and all derivatives is the same as the space considered in Conjecture 5.1.1.

Proof. To begin with, one can show that the algebra generated by the operators

$$
\begin{aligned}
E_{h, k}^{0} & =\sum_{i=1}^{n} y_{i} \partial x_{i}^{h} \partial y_{i}^{k} \\
E_{h, k}^{1} & =\sum_{i=1}^{n} x_{i} \partial x_{i}^{h} \partial y_{i}^{k}
\end{aligned}
$$


together with the operators $P_{h, k}$ of (114) is the same as that generated by the $P_{h, k}$ and all operators of the form (117). Since the $P_{h, k}$ kill all harmonics, we need only apply to $\Delta(X)$ products of operators of the form (118) and pure differential operators. The commutator of two operators (118) is again a linear combination of such operators, and the commutator of an operator (118) with a pure differential operator is pure differential. Thus the space $L$ spanned by pure differential operators and operators (118) is a Lie algebra which generates the algebra of operators we intend to apply to $\Delta(X)$.

By the Poincaré-Birkhoff-Witt theorem it suffices to consider operator products

$$
p(\partial X)\left(E_{1,0}^{0}\right)^{m_{0,1,0}} \cdots\left(E_{M, N}^{1}\right)^{m_{1, M, N}} q(\partial Y) \Delta(X),
$$

where $p(\partial X)$ is a monomial in the $\partial x_{i}, q(\partial Y)$ is a monomial in the $\partial y_{i}$, and the order in which the factors involving $E_{h, k}^{i}$ appear is fixed in some suitably chosen way. In particular, we may put any factors $E_{h, k}^{i}$ with $k>0$ or $h>n-1$ and any factors $E_{h, 0}^{1}$ after all other factors. But these factors, as well as $q(\partial Y)$, represent operators which either kill $\Delta(X)$ or have $\Delta(X)$ as an eigenfunction; thus we may omit them entirely, and consider only products

$$
p(\partial X)\left(E_{1,0}^{0}\right)^{m_{1}} \cdots\left(E_{n-1,0}^{0}\right)^{m_{n-1}} \Delta(X) .
$$

Since $E_{p, 0}^{0}$ is the same as $E_{p}$, the proof is complete.

Among other things, the above proposition implies that the operator conjecture is more symmetric than it first appears.

COROLlaRY 5.2.1. The space generated by the operators $E_{p}$ and $\partial x_{i}$ applied to $\Delta(X)$ is the same as the space generated by the operators $F_{p}$ and $\partial y_{i}$ applied to $\Delta(Y)$, where $F_{p}$ is $E_{p}$ with $x$ and $y$ interchanged.

Proof. The first space contains $\Delta(Y)$, since apart from a scalar factor $\Delta(Y)=$ $E_{1}^{\left(\frac{n}{2}\right)} \Delta(X)$. By Proposition 5.2.1 the first space is closed under the operators generating the second space and hence contains the second space. By symmetry the second space also contains the first.

\section{3. $\phi_{a}$ approach}

An alternative statement of the operator conjecture would say that the ideal $I$ defining $R_{n}$ coincides with the orthogonal complement $J$ of the space considered in the conjecture. The ideal $J$ admits the following nice description which may be useful for studying the conjecture.

Proposition 5.3.1. The ideal $J$ orthogonal to the space considered in Conjecture 5.1 .1 consists of all $f(X, Y)$ such that 


$$
f\left(x_{1}, \ldots, x_{n}, \phi_{a}\left(x_{1}\right), \ldots, \phi_{a}\left(x_{n}\right)\right) \in I
$$

for all polynomials $\phi_{a}(x)=a_{1} x+a_{2} x^{2}+\cdots+a_{n-1} x^{n-1}$.

Note that there are no $y$ 's in (121) so the $I$ appearing there may be understood as the classical ideal generated by symmetric polynomials in $\mathbb{Q}[X]$. The operator conjecture then asserts that $f(X, Y) \in I$ if and only if $f\left(X, \phi_{a}(X)\right)$ belongs to this classical ideal for all values of the coefficients $a$.

Proof of Proposition 5.3.1. Recall that multiplication by $x_{i}$ is adjoint to $\partial x_{i}$, so the adjoint of $E_{p}$ is given by

$$
E_{p}^{*}=\sum_{i=1}^{n} x_{i}^{p} \partial y_{i}
$$

Therefore $f(X, Y)$ belongs to $J$ if and only if for all $e_{1}, \ldots, e_{n-1},\left(E_{1}^{*}\right)^{e_{1}} \ldots$ $\left(E_{n-1}^{*}\right)^{e_{n-1} f}$ is orthogonal to $\Delta(X)$ and its derivatives, that is, if and only if $\left.\left(E_{1}^{*}\right)^{e_{1}} \cdots\left(E_{n-1}^{*}\right)^{e_{n-1}} f\right|_{Y=0} \in I$. Now $\left(E_{1}^{*}\right)^{e_{1}} \cdots\left(E_{n-1}^{*}\right)^{e_{n-1} f}$ is the coefficient of $a_{1}^{e_{1}} \cdots a_{n-1}^{e_{n-1}} / e_{1} ! \cdots e_{n-1} !$ in $\exp \left(a_{1} E_{1}^{*}+\cdots+a_{n-1} E_{n-1}^{*}\right) f$, and this last expression is equal to $f\left(x_{1}, \ldots, x_{n}, y_{1}+\phi_{a}\left(x_{1}\right), \ldots, y_{n}+\phi_{a}\left(x_{n}\right)\right)$, since $\exp \left(E_{p}^{*}\right)$ is the operator translating $y_{i}$ by $x_{i}^{p}$. Setting all $y_{i}=0$, the result follows.

Notes. Proposition 5.3.1 is a joint result of Garsia and myself.

\subsection{Consequences: socle; monomials in $I ; \prod_{i}\left(\alpha_{i} x_{i}+\beta_{i} y_{i}\right)$}

The operator conjecture has some implications which might be of interest to prove in and of themselves. I will discuss a couple of these, which have not been proven yet.

Definition. The socle of $R_{n}$ (or more generally of any module over a polynomial ring) consists of those elements annihilated by all $x_{i}$ and $y_{i}$.

An important consequence of the operator conjecture for the structure of the ring $R_{n}$ is the following.

Conjecture 5.4.1. The socle of $R_{n}$ consists exactly of its alternating polynomials.

The fact that the operator conjecture implies this is easy to deduce using the adjointness of differentiation and multiplication.

Another consequence of the operator conjecture is that it determines exactly which monomials in $\mathbb{Q}[X, Y]$ belong to the ideal $I$. They are just the monomials which do not appear in any diagonal harmonic. By the operator conjecture 
the monomials that do appear in some diagonal harmonic are exactly those obtained from monomials appearing in the Vandermonde determinant $\Delta(X)$ by replacing $x_{i}^{l}$ with some $x_{i}^{h} y_{i}^{k}$ for each $i$, where $h+k \leq l$. But the monomials appearing in $\Delta(X)$ are simply permutations of $x_{1}^{0} x_{2}^{1} \cdots x_{n}^{n-1}$, so we arrive at the following restatement.

Conjecture 5.4.2. A monomial belongs to the ideal $I$ if and only if for some $k$ there are more than $n-k$ indices $i$ for which the exponents of $x_{i}$ and $y_{i}$ total at least $k$.

As a particular case, when $k=1$ this says monomials involving every index $i$ with nonzero exponent belong to $I$, or in other words

$$
\prod_{i=1}^{n}\left(\alpha_{i} x_{i}+\beta_{i} y_{i}\right) \in I
$$

The above identity has been proven by J. Alfano, using an explicit straightening technique in the spirit of the results in Section 3.2. The full conjecture remains open at present.

\subsection{Relation to the conjectures for $q=t^{-1}$}

Assuming the operator conjecture, we can relate the Frobenius series of the harmonics $H_{n}$ and the Hilbert series of its alternating part to two new commutative rings arising from the application of operators to the Vandermonde $\Delta(X)$. This is done as follows.

Definition. Let $J$ be the ideal in $\mathbb{Q}\left[x_{1}, \ldots, x_{n}, e_{1}, \ldots, e_{n-1}\right]$ consisting of all polynomials $p(X, E)$ such that the operator $p\left(\partial x_{1}, \ldots, \partial x_{n}, E_{1}, \ldots, E_{n-1}\right)$ kills $\Delta(X)$. Define rings

$$
T_{n}=\mathbb{Q}\left[x_{1}, \ldots, x_{n}, e_{1}, \ldots, e_{n-1}\right] / J
$$

and

$$
U_{n}=\mathbb{Q}\left[e_{1}, \ldots, e_{n-1}\right] /(J \cap \mathbb{Q}[E]) .
$$

These rings are $S_{n}$ modules, where we let $S_{n}$ permute the $x_{i}$ and fix the $e_{k}$. They are also doubly graded if we assign degree $(1,0)$ to each $x_{i}$ and degree $(k, 1)$ to each $e_{k}$. The operator conjecture then gives the Frobenius series in terms of that of $H_{n}$, as follows.

Conjecture 5.5.1. The Frobenius series of $T_{n}$ is $t_{2}^{\left(\begin{array}{l}n \\ 2\end{array}\right)} \omega \mathcal{F}_{n}\left(t^{-1}, q\right)$, where $\omega$ is the canonical involution on symmetric functions corresponding to tensoring with 
the sign character. The Hilbert series of $U_{n}$ is $t^{\left(\frac{n}{3}\right)} C_{n}\left(t^{-1}, q\right)$, where $C_{n}$ is the conjectured $t, q$-Catalan number defined by (43).

The rings $T_{n}$ and $U_{n}$ can be given a direct description, which is at least superficially independent of their definition in terms of operators.

Proposition 5.5.1. Let $N$ be the ring $\mathbb{Q}[X, Y] /\left(p_{1}(X), \ldots, p_{n}(X)\right)$, where $p_{i}(X)$ are the power sums (or any other set of fundamental invariants for $S_{n}$ ). Then $T_{n}$ is isomorphic to the subring of $N$ generated by the $x_{i}$ and $e_{k}=\sum_{i} y_{i} x_{i}^{k}$, and $U_{n}$ is isomorphic to the subring generated by the $e_{k}$ alone.

Proof. An operator which is a polynomial in $y_{i}$ and $\partial x_{i}$ kills $\Delta(X)$ if and only if its coefficient of each $y$ monomial belongs to the ideal in $\mathbb{Q}[x]$ generated by the $p_{k}(X)$, which is to say, if and only if the corresponding polynomial in $y_{i}$ and $x_{i}$ vanishes in $N$. Since the $e_{k}$ in the statement correspond to the operators $E_{k}$ and the $x_{i}$ correspond to the $\partial x_{i}$, the result follows immediately.

An interesting fact is that for the single grading of $U_{n}$ and $T_{n}$ by total degree (corresponding to the $q=t^{-1}$ specialization for $H_{n}$ ), the conjectured Frobenius series resemble those of polynomial rings modulo ideals generated by homogeneous systems of parameters.

PROPOSITION 5.5.2. Suppose $\mathbb{U}_{n}=\mathbb{Q}\left[e_{1}, \ldots, e_{n-1}\right] /\left(\theta_{1}, \ldots, \theta_{n-1}\right)$, where the degree of $e_{k}$ is defined to be $k+1$, and $\left(\theta_{i}\right)$ is an h.s.o.p. with $\theta_{i}$ of degree $n+i+1$. Then the Hilbert series of $\bar{O}_{n}$ is the one conjectured for $U_{n}$ i.e., it is given by (45).

PROPOSITION 5.5.3. Suppose $T_{n}=\mathbb{Q}\left[x_{1}, \ldots, x_{n}, e_{1}, \ldots, e_{n-1}\right] /\left(\theta_{1}, \ldots, \theta_{2 n-1}\right)$, where $x_{i}$ has degree $1, e_{k}$ has degree $k+1,\left(\theta_{i}\right)$ is an h.s.o.p., $\theta_{1}, \ldots, \theta_{n}$ are $S_{n}$ invariants of degrees 1 through $n$, and $\theta_{n+1}, \ldots, \theta_{2 n-1}$ are of degree $n+1$ and span an irreducible reflection representation of $S_{n}$. Then the Frobenius series of $T_{n}$ is the one conjectured for $T_{n}$ i.e., it is given by $\omega$ applied to (30).

Proofs. Proposition 5.5.2 is immediate - just write (45) as

$$
\frac{\left(1-q^{n+2}\right) \cdots\left(1-q^{2 n}\right)}{\left(1-q^{2}\right) \cdots\left(1-q^{n}\right)}
$$

Proposition 5.5.3 follows from Proposition 2.5.2, since the contribution of the invariant variables $e_{1}, \ldots, e_{n-1}$ exactly cancels that of the invariant parameters $\theta_{2}, \ldots, \theta_{n}$.

One might hope that the rings $T_{n}$ and $U_{n}$ themselves are defined by h.s.o.p.s in the manner of $T_{n}$ and $O_{n}$ (that is, are complete intersection rings). Unfortunately, this is false; neither ring is even Gorenstein for $n>2$. Nevertheless it is 
possible that the ideals defining $T_{n}$ and $U_{n}$ occur via some sort of grading of nonbihomogeneous ideals defining rings of the form $\widetilde{T}_{n}$ and $\tilde{U}_{n}$.

\section{Geometric interpretation}

Let $M_{n}$ be the set of $n \times n$ matrices, considered as an algebraic variety; its coordinate ring consists of all polynomial functions in the entries $x_{i, j}$ of the general matrix $X \in M_{n}$. We let $G L_{n}$ act on $M_{n}$ by conjugation: $g$ sends $X$ to $X^{g}=g^{-1} X g$. The polynomials $p_{k}(X)=\operatorname{tr}\left(X^{k}\right)$ are $G L_{n}$ invariant. Restricted to the diagonal matrices $X=\operatorname{diag}\left(x_{1}, \ldots, x_{n}\right)$ they become the power sums $p_{k}\left(x_{1}, \ldots, x_{n}\right)$, which are fundamental invariants for the reflection action of $S_{n}$.

The polynomials $p_{k}(X)$ vanish when the matrix $X$ is nilpotent, and it turns out that they generate the ideal $I(\mathcal{N})$ of all polynomials vanishing on the variety $\mathcal{N} \subseteq M_{n}$ of nilpotent matrices. By the celebrated results of Kostant [15], analogous statements hold when $G L_{n}$ is replaced by any semisimple complex Lie group, $M_{n}$ by its Lie algebra, $\mathcal{N}$ by the set of nilpotent elements in the Lie algebra, the diagonal matrices by a Cartan subalgebra, and $S_{n}$ by the Weyl group.

In this section we consider a geometrical setup parallel to the classical one just described which relates $R_{n}$ to the variety of pairs of commuting nilpotent matrices. All the material in this section grows out of discussions with $\mathbf{H}$. Kraft.

\subsection{The commuting variety}

Definition. The commuting variety $\mathcal{C}$ is the subvariety of $M_{n} \times M_{n}$ consisting of pairs of matrices $(X, Y)$ such that $X Y=Y X$.

Remarkably little is known about the commuting variety. It is not even known whether the defining equations $X Y=Y X$ generate its ideal. (This is really a system of $n^{2}$ equations, one for each matrix entry.) Hochster has raised the question whether the commuting variety is Cohen-Macaulay. This is known to be true for $n \leq 3$.

At present, the only nontrivial theorem about the commuting variety is the following.

Proposition 6.1.1 (Gerstenhaber [9]). The commuting variety is irreducible, of dimension $n^{2}+n$

Proposition 6.1.1 is equivalent to the statement that the set of pairs $(X, Y)$ with $X$ and $Y$ regular semisimple is dense in $\mathcal{C}$. This means $X$ and $Y$ are conjugates (by the same $g$ ) of diagonal matrices with distinct eigenvalues. The statement is equivalent to the proposition because the set in question is easily seen to be open, irreducible, and of the correct dimension. 


\subsection{Joint eigenvalues and diagonal action of $S_{n}$}

Let $\mathfrak{h}$ denote the set of diagonal matrices. Since diagonal matrices commute with each other, we have $\mathfrak{h} \times \mathfrak{h} \subseteq \mathcal{C} \subseteq M_{n} \times M_{n}$. Writing elements of $\mathfrak{h} \times \mathfrak{h}$ as $\left(\operatorname{diag}\left(x_{1}, \ldots, x_{n}\right), \operatorname{diag}\left(y_{1}, \ldots, y_{n}\right)\right)$, we identify the coordinate ring of $\mathfrak{h} \times \mathfrak{h}$ with $\mathbb{Q}[X, Y]=\mathbb{Q}\left[x_{1}, \ldots, x_{n}, y_{1}, \ldots, y_{n}\right]$.

Any $G L_{n}$ invariant polynomial function on $\mathcal{C}$ restricts to an $S_{n}$ invariant polynomial on $\mathfrak{h} \times \mathfrak{h}$ since the action of $S_{n}$ on diagonal matrices can be realized by conjugating with permutation matrices $g$.

Proposition 6.1.1 has the following consequence.

PROPOSITION 6.2.1. The map $\phi$ sending each $G L_{n}$ invariant polynomial on $\mathcal{C}$ to its restriction to $\mathfrak{h} \times \mathfrak{h}$ is an isomorphism from $\mathcal{O}(\mathcal{C})^{G L_{n}}$ to $\mathbb{Q}[X, Y]^{S_{n}}$. Here $\mathcal{O}(\mathcal{C})$ denotes the coordinate ring of $\mathcal{C}$, and $\mathcal{O}(\mathcal{C})^{G L_{n}}$ its subring of $G L_{n}$ invariants.

Proof. First, we show the map $\phi$ is injective. By Proposition 6.1.1, a function on $\mathcal{C}$ is determined by its values on conjugates of pairs of diagonal matrices. If the function is $G L_{n}$ invariant, it is therefore determined by its values on diagonal matrices themselves, that is, by its restriction to $\mathfrak{h} \times \mathfrak{h}$.

Second, we show that $\phi$ is surjective. By Proposition 1.2.1, $\mathbb{Q}[X, Y]^{S_{n}}$ is generated by the power sums $p_{h, k}=\sum_{i} x_{i}^{h} y_{i}^{k}$. But $p_{h, k}$ is the restriction to $\mathfrak{h} \times \mathfrak{h}$ of the $G L_{n}$ invariant polynomial $\operatorname{tr}\left(X^{h} Y^{k}\right)$ on $C$.

Remark. Richardson [20] shows that Proposition 6.1.1 holds with $G L_{n}$ replaced by any semisimple complex Lie group. Proposition 6.2.1 is also conjectured to hold in all cases. For types $G_{2}$ and $B_{n}$, essentially the same proof applies. For types $F_{4}$ and $D_{n}$, Weyl's theorem (Proposition 1.2.1) fails, but Wallach [28] has been able to obtain the result using something weaker. The conjecture remains unproven for types $E_{6}, E_{7}$, and $E_{8}$.

It is clear from the preceding discussion that when $X$ and $Y$ commute the function $\operatorname{tr}\left(X^{h} Y^{k}\right)$ is the polarized power sum $p_{h, k}\left(\alpha_{1}, \ldots, \alpha_{n}, \beta_{1}, \ldots, \beta_{n}\right)$ where $\left\{\left(\alpha_{i}, \beta_{i}\right)\right\}$ are the joint eigenvalues of $X$ and $Y$. Thus Proposition 6.2.1 says that the $G L_{n}$ invariant polynomials on $\mathcal{C}$ are precisely the symmetric polynomials (under the diagonal action of $S_{n}$ ) in the joint eigenvalues of $X$ and $Y$.

\section{3. $R_{n}=$ commuting nilpotents $\cap$ diagonal}

Since $X$ and $Y$ are both nilpotent just in case their joint eigenvalues are all zero, we have immediately the following corollary to Proposition 6.2.1.

COROLLARY 6.3.1. The restriction to $\mathfrak{h} \times \mathfrak{h}$ of the ideal generated by $G L_{n}$ invariant functions on $\mathcal{C}$ which vanish on the commuting nullcone $(\mathcal{N} \times \mathcal{N}) \cap \mathcal{C}$ is the ideal $I_{n}$ defining $R_{n}$. 
We conjecture, but do not know how to prove, that the ideal $I((\mathcal{N} \times \mathcal{N}) \cap \mathcal{C})$ is generated by its $G L_{n}$ invariant elements, that is, by polynomials $\operatorname{tr}\left(X^{h} Y^{k}\right)$ for $h+k>0$. Then we have:

COROLLARY 6.3.2. If the ideal of the commuting nullcone (as a subvariety of the commuting variety) is generated by $G L_{n}$ invariants, then the restriction of this ideal to $\mathfrak{h} \times \mathfrak{h}$ is $I_{n}$.

The conclusion may also be phrased geometrically: Both the commuting nullcone and $\mathfrak{h} \times \mathfrak{h}$ are subvarieties of $\mathcal{C}$. Set-theoretically, their intersection is $\{(0,0)\}$ since a nilpotent diagonal matrix must be zero. But their intersection as subschemes of $\mathcal{C}$ is by definition the subscheme defined by the sum of their defining ideals. The conclusion of Corollary 6.3.2 amounts to the assertion that $R_{n}$ is the coordinate ring of this subscheme.

Although the conjecture that the ideal of the commuting nullcone is generated by $G L_{n}$ invariants seems as hard to prove as anything else about the commuting variety, it turns out that for the consequence of it given by Corollary 6.3 .2 , the operator conjecture suffices.

PROPOSITION 6.3.1. If the operator conjecture holds, then the restriction of the ideal of the commuting nullcone to $\mathfrak{h} \times \mathfrak{h}$ is $I_{n}$.

Proof. Consider the following three ideals in $\mathbb{Q}[X, Y]$ :

$$
J_{1}=\left\{f\left(\partial x_{i}, E_{k}\right) \Delta(X)\right\}^{\perp}
$$

the orthogonal complement of the space of harmonics figuring in the operator conjecture,

$$
J_{2}=\left.I((\mathcal{N} \times \mathcal{N}) \cap \mathcal{C})\right|_{\mathfrak{h} \times \mathfrak{h}}
$$

and

$$
J_{3}=I_{n} .
$$

We will prove

$$
J_{1} \supseteq J_{2} \supseteq J_{3} .
$$

Since the operator conjecture is $J_{1}=J_{3}$ it follows that all three ideals are the same if it holds.

We have already seen that the polarized power sums $p_{h, k}$ are restrictions of polynomials vanishing on $(\mathcal{N} \times \mathcal{N}) \cap \mathcal{C}$, so $J_{2} \supseteq J_{3}$. The problem is to show $J_{2} \subseteq J_{1}$.

If $f(X, Y)$ belongs to $J_{2}$ there is a matrix polynomial $g(X, Y)$ which vanishes on the commuting nullcone and restricts to $f$ on $\mathfrak{h} \times \mathfrak{h}$. If $X$ is a nilpotent matrix, 
then $\phi_{a}(X)$ is a nilpotent matrix commuting with $X$, so $\left(X, \phi_{a}(X)\right) \in(\mathcal{N} \times \mathcal{N}) \cap \mathcal{C}$ and $g\left(X, \phi_{a}(X)\right)=0$ for all polynomials $\phi_{a}(z)=a_{1} z+\cdots+a_{n-1} z^{n-1}$.

As we noted in the introductory remarks to this section, a matrix polynomial $h(X)$ which vanishes on $\mathcal{N}$ belongs to the ideal generated by the polynomials $\operatorname{tr}\left(X^{k}\right)$. In particular this is true of $g\left(X, \phi_{a}(X)\right)$. Restricting to $\mathfrak{h} \times \mathfrak{h}$ we find that $f\left(X, \phi_{a}(X)\right) \in\left(p_{1}, \ldots, p_{n}\right)$, hence $f \in J_{1}$ by Proposition 5.3.1.

\section{Other Coxeter and Weyl groups}

In this section we consider analogs of the ring $R_{n}$ and our conjectures about it for general reflection groups. Data from the computer suggest how the conjectures should extend, but a curious phenomenon occurs - the data do not actually satisfy the extended conjectures, but only approximate them. Below we indicate in detail what happens and speculate somewhat on a possible explanation.

Throughout what follows I assume considerable familiarity with the theory of reflection groups. A good general reference is the recent book by Humphreys [13].

\subsection{Analogs of $2.1,2.2$}

Let $W$ be a reflection group, acting by its irreducible reflection representation on a vector space $U$. The coordinate ring $\mathbb{Q}[U \oplus U]$ carries an action of $W$ which generalizes our diagonal action of $S_{n}$. The dimension of $U$ is called the rank of $W$, which for $S_{n}$ is $n-1 . \quad\left(S_{n}\right.$ is usually referred to in this context as the reflection group of type $A_{n-1}$, using a subscript that corresponds to the rank.) There is a $W$ invariant h.s.o.p. in $\mathbb{Q}[U]$ whose elements generate all the $W$ invariants. Such an h.s.o.p. is called a set of fundamental invariants. The degrees of fundamental invariants are uniquely determined by $W$; the numbers which are 1 less than the degrees are called the exponents. The largest degree is called the Coxeter number.

In terms of the above statistics, the value $(n+1)^{n-1}$ proposed by Conjecture 2.1.1 for the dimension of $R_{n}$ can be expressed as $(h+1)^{\ell}$ where $h$ is the Coxeter number and $\ell$ is the rank. As we shall see below there are reasons to expect the quantity $(h+1)^{\ell}$ to possess special significance, at least for Weyl groups. A little wishful thinking then suggests the following.

Guess 7.1.1. (Incorrect, but see 7.1.1). The quotient of $Q[U \oplus U]$ by the ideal generated by $W$ invariants without constant term has dimension $(h+1)^{\ell}$.

Guess 7.1.1 is correct for the dihedral groups (see Section 7.5) and for $B_{3}$. For $S_{n}$ it is Conjecture 2.1.1. The following groups $W$ are counterexamples, but as the table indicates, they appear to be the proverbial "exceptions that prove the rule." 


\begin{tabular}{c|c|c} 
Group & $(h+1)^{\ell}$ & Actual dimension \\
\hline$B_{4}$ & $9^{4}$ & $9^{4}+1$ \\
$B_{5}$ & $11^{5}$ & $11^{5}+33$ \\
$D_{4}$ & $7^{4}$ & $7^{4}+40$
\end{tabular}

Since the numbers seem too striking to be mere coincidence, we propose the following genuine conjecture.

Conjecture 7.1.1. For each reflection group $W$, or at least for each Weyl group, there is a "natural" quotient ring $R_{W}$ of $\mathbb{Q}[U \oplus U]$ by some doubly homogenous ideal containing the invariants without constant term, such that $\operatorname{dim}\left(R_{W}\right)=$ $(h+1)^{\ell}$. The ideal should be $\boldsymbol{s l}_{2}$ invariant and its restriction to $\mathbb{Q}[U]$ should be the classical one generated by the fundamental invariants.

For $B_{4}, B_{5}$, and $D_{4}$ suitable ideals do exist. Computations by J. Alfano show that they can be chosen (uniquely, in these cases) so that the rings $R_{W}$ satisfy further conjectures, including the following analog of Conjecture 2.2.1.

Conjecture 7.1.2. The natural $R_{W}$ of Conjecture 7.1.1. has Hilbert series $\mathcal{H}_{W}(t, q)$ satisfying

$$
q^{h / 2} \mathcal{H}_{w}\left(q^{-1}, q\right)=\left([h+1]_{q}\right)^{\ell}=\left(1+q+\cdots+q^{h}\right)^{\ell} .
$$

Note that $h \ell / 2$ is an integer - it is the degree of the discriminant $\Delta_{W}$, the sum of the exponents, and the number of reflections in $W$.

\subsection{Failure of operator conjecture for $B_{4}$}

The operators $E_{p}$ defined by (115) can be given an intrinsic definition which applies to any reflection group.

For this we first introduce a $W$ invariant positive definite form $\langle\cdot, \cdot\rangle$ on $U$. Since $U$ is irreducible, the form is unique up to a scalar multiple. It extends to a $W$ invariant apolar form on $\mathbb{Q}[U \oplus U]$ as explained at the end of Section 1.3.

Let $x_{1}, \ldots, x_{\ell}$ be a basis of $U$, and let $y_{1}, \ldots, y_{\ell}$ be the dual basis, i.e., $\left\langle x_{i}, y_{j}\right\rangle=\delta_{i j}$ for all $i, j$. We regard the ring $Q[U \oplus U]$ as a polynomial ring $\mathbb{Q}\left[x_{1}, \ldots, x_{\ell}, y_{1}, \ldots, y_{\ell}\right]$, using the basis $\left\{x_{i}\right\}$ for the first summand of $U \oplus U$ and $\left\{y_{i}\right\}$ for the second.

Note that the derivations $\partial y_{i}$, which by definition satisfy $\partial y_{i}\left(y_{j}\right)=\delta_{i j}$, transform dually to the $y_{i}$ under change of basis. In other words, they transform the same as the $x_{i}$ and the same as the differential forms $d x_{i}$.

Now let $p_{1}, \ldots, p_{\ell}$ be a system of fundamental invariants for $W$, written as polynomials in the $x_{i}$. Their differentials $d p_{k}$ have unique expansions 


$$
d p_{k}=\sum_{i=1}^{\ell} \frac{\partial p_{k}}{\partial x_{i}} d x_{i},
$$

and we define operators $E_{k}^{*}$ by substituting $\partial y_{i}$ for $d x_{i}$ :

$$
E_{k}^{*}=\sum_{i=1}^{\ell} \frac{\partial p_{k}}{\partial x_{i}} \partial y_{i} .
$$

By the remarks made above, this definition is independent of the choice of basis $x_{i}$. In particular this implies the operators $E_{k}^{*}$ are $W$ invariant. Finally we define $E_{k}$ to be the adjoint of $E_{k}^{*}$ with respect to the apolar form. Thus $E_{k}$ is an invariant operator, linear in the $y_{i}$ and polynomial in the $\partial x_{i}$, of bidegree $\left(-e_{k}, 1\right)$ where $e_{k}$ is the exponent $\operatorname{deg}\left(p_{k}\right)-1$. One proves just as in Section 5.1 that these operators carry the space of harmonics into itself.

For the case $W=S_{n}$ it is easy to see that this construction agrees with the definition (5.1.2) of $E_{k}$.

The analog of the operator conjecture 5.1.1 would now be the following.

Guess 7.2.1. (Incorrect but see Fact 7.2.1. and Conjecture 7.2.1). Let $\mathcal{H}_{W}$ be the space of diagonal harmonics for $W$, i.e., the orthogonal complement under the invariant apolar form of the ideal generated by invariants without constant term in $\mathbb{Q}[U \oplus U]$. Then $\mathcal{H}_{W}$ is the smallest space containing the discriminant $\Delta(X)$ and closed under the action of the operators $\partial x_{i}$ and $E_{1}, \ldots, E_{\ell}$.

A counterexample to this is $W=B_{4}$, but again it seems to be an exception that proves the rule, because of the following.

Fact 7.2.1. For $W=B_{4}$, the space of harmonics generated by the operators $E_{1}, \ldots, E_{4}$ and the $\partial x_{i}$ applied to the discriminant $\Delta(X)$ has dimension $9^{4}-1$.

Recall the proof of Proposition 6.3, in which we considered three ideals: $J_{1}$, the ideal orthogonal to the space of harmonics considered in the operator conjecture; $J_{2}$, the restriction to $\mathfrak{h} \times \mathfrak{h}$ of the ideal of the commuting nullcone; and $J_{3}$, the ideal generated by diagonal invariants without constant term. The proof that $J_{1} \supseteq J_{2} \supseteq J_{3}$ generalizes to any semisimple complex Lie algebra and its Weyl group.

In the case of $B_{4}$, the dimension of the quotient by $J_{1}$ is $9^{4}-1$, while the dimension of the quotient by $J_{3}$ is $9^{4}+1$. We make the following conjecture, admittedly on the basis of little more than hope for justice in the mathematical universe.

Conjecture 7.2.1. Let $\mathfrak{g}$ be a semisimple complex Lie algebra, $\mathfrak{h}$ a Cartan subalgebra, and $W$ the Weyl group. Let $\mathcal{C}_{\mathcal{N}}$ be the commuting nullcone, i.e., the set of pairs $(X, Y) \in \mathfrak{g} \times \mathfrak{g}$ such that $X$ and $Y$ commute and are nilpotent. Let $I_{W}$ 
be the restriction to $\mathbb{Q}[U \oplus U]$ (which is the coordinate ring of $\mathfrak{h} \times \mathfrak{h}$ ) of $I\left(\mathcal{C}_{\mathcal{N}}\right)$. Then $R_{W}=\mathbb{Q}[U \oplus U] / I_{W}$ is the natural quotient ring satisfying Conjectures 7.1.1 and 7.1.2.

For clarity, let us work out the example $W=B_{4}$ in a little more detail. The group $B_{4}$ is the group of signed permutations of four letters. It acts diagonally on $\mathbb{Q}\left[x_{1}, \ldots, x_{4}, y_{1}, \ldots, y_{4}\right]$ in the obvious way. The invariants are the $S_{4}$ invariants whose monomials are of even total degree in $x_{i}$ and $y_{i}$ for each $i$, so that the simultaneous sign change $x_{i} \mapsto-x_{i}, y_{i} \mapsto-y_{i}$ has no effect. They are generated by the polarized power sums $p_{h, k}(X, Y)$ for $h+k$ even. The discriminant $\Delta(X)$ is the product of the variables times the Vandermonde determinant in their squares:

$$
\Delta(X)=x_{1} \cdots x_{n} \prod_{i<j}\left(x_{i}^{2}-x_{j}^{2}\right) .
$$

The operators $E_{k}$ are the operators $E_{p}$ given by (115) for $p=1,3,5,7$. The analog of Proposition 5.5.1 holds, with the same proof; this is how the dimension $9^{4}-1$ for the space of harmonics appearing in the operator conjecture was computed.

We may take $\mathfrak{g}$ to be the Lie algebra $0_{9}$ of skew-symmetric $9 \times 9$ matrices (alternatively, the Lie algebra $\mathfrak{s p}_{8}$ has the same Weyl group, but its description is more complicated). The commuting nullcone consists of pairs of such matrices which commute with each other and are nilpotent. The Cartan subalgebra $b$ can be taken to consist of the antidiagonal matrices

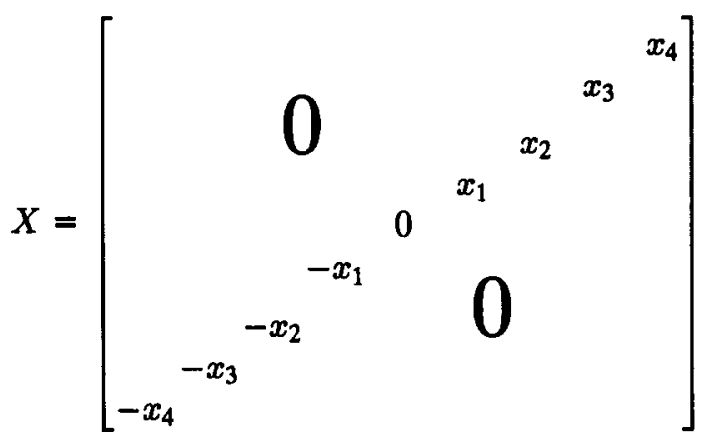

Thus $J_{2}$ will be the ideal of polynomials in $\mathbb{Q}\left[x_{1}, \ldots, x_{4}, y_{1}, \ldots, y_{4}\right]$ obtained by restricting to pairs $(X, Y)$ of matrices of the form (136) arbitrary matrix polynomials which vanish on all pairs of commuting skew-symmetric $9 \times 9$ matrices. In particular, this includes the matrix polynomials $\operatorname{tr}\left(X^{h} Y^{k}\right)$, whose restriction to $\mathfrak{h} \times \mathfrak{h}$ is $p_{h, k}$ if $h+k$ is even, and zero if $h+k$ is odd. In this way we see explicitly how $J_{3} \subseteq J_{2}$.

If Conjecture 7.2.1 is correct, there must be an "extra" polynomial vanishing on all pairs of commuting skew-symmetric $9 \times 9$ matrices, whose restriction to pairs of the form (136) is the extra element of $J_{2}$, modulo $J_{3}$. By Alfano's 
computations, we know what this restriction must be: modulo the $B_{4}$ diagonal invariants it is

$$
\sum_{i=1}^{4} y_{i}^{3} \prod_{j \neq i} x_{j} .
$$

This polynomial is interesting for another reason. It represents the sole $D_{4}$ diagonal invariant not in the ideal generated by polarizations of the fundamental invariants.

Unfortunately, it is infeasible to compute the ideal of the commuting nullcone for $\mathfrak{s o}_{9}$ by brute force, so for the moment there is little hope of testing the $B_{4}$ case of Conjecture 7.2.1 by direct computation.

\subsection{Reduction of the root lattice (compare 2.4)}

What we wish to do next is establish, when $W$ is a Weyl group, an analog of Conjecture 2.4.1 for the putative $R_{W}$ satisfying Conjectures 7.1.1 and 7.1.2. We are unavoidably on shaky ground here, since even our proposed definition of $R_{W}$ in Conjecture 7.2.1 is uncertain.

Nevertheless, we have explicit candidates for $R_{W}$ in the cases $B_{4}, B_{5}$, and $D_{4}$ from Alfano's computations, and for $B_{2}$ and $B_{3}$ the quotient ring by diagonal invariants works as $R_{W}$. In these cases all our conjectures hold for the proposed $R_{W}$ (except that we are unable to determine whether this $R_{W}$ is the one given by Conjecture 7.2.1). In addition, although what we do makes sense only for Weyl groups, it leads to predictions that make sense for arbitrary Coxeter groups, and these predictions have been verified for the dihedral groups.

Also, as we shall see, the process of deriving predictions that tend to confirm the conjecture leads to surprising and beautiful formulas whose very unexpectedness supports the idea that the conjecture is somehow fundamentally natural.

In this section and the following ones I assume familiarity with Weyl groups and affine Weyl groups, roots, weights, the fundamental alcove, and so on. The following conjecture reduces to Conjecture 2.4.1 when $W=S_{n}$.

Conjecture 7.3.1. Let $W$ be a Weyl group with coxeter number $h, Q$ its root lattice, and $Y$ the permutation representation of $W$ on elements of the finite group $Q / p Q$, where $p=h+1$. Then the representation of $W$ on the natural $R_{W}$ of Conjecture 7.1.1 is isomorphic to $\varepsilon \otimes Y$, where $\varepsilon$ is the sign representation.

Note that this conjecture implies Conjecture 7.1.1., since $Q / p Q$ has $(h+l)^{\ell}$ elements.

Given the way Conjecture 2.4.1 is stated, it might seem more natural to replace the root lattice $Q$ with the weight lattice $\Lambda$ in Conjecture 7.3.1. The following proposition shows that the choice doesn't matter as far as the conclusions are 
concerned. For technical reasons, however, the use of the root lattice will be essential in the next section.

Proposition 7.3.1. Let $W$ be a Weyl group, $Q$ the root lattice, and $\Lambda^{\vee}$ the coweight lattice. Then for any $p$ the permutation representation of $W$ on $\Lambda^{\vee} / p \Lambda^{\vee}$ has the same character as the representation on $Q / p Q$.

Proof. The character of the permutation representation at $w \in W$ is given by the number of fixed points of $W$.

By definition we have $\left(\lambda_{i}^{V}, \alpha_{j}\right)=\delta_{i j}$ for the fundamental weights $\lambda_{i}$ and simple roots $\alpha_{j}$. Thus the matrix entries of $w$ with respect to the basis $\left\{\alpha_{i}\right\}$ are given by $\left(\lambda_{i}^{\vee}, w \alpha_{j}\right)$. This is the same as $\left(w^{-1} \lambda_{i}^{\vee}, \alpha_{j}\right)$, so the matrix of $w$ on $\Lambda^{\vee}$ is $\left(M_{w}^{-1}\right)^{T}$, where $M_{w}$ is the matrix of $w$ on $Q$.

The fixed points of $w$ in $Q / p Q$ correspond to solutions modulo $p$ of the equation $\left(M_{w}-I\right) x=0$. We claim that for any square integer matrix $A$ the equations $A x=0$ and $A^{T} x=0$ have the same number of solutions modulo $p$, which clearly implies the result.

For the claim, observe that the number of solutions is unchanged when $A$ is multiplied on either the left or right by any integer matrix with unit determinant. Hence we are free to replace $A$ by its Smith canonical form, which is a diagonal matrix.

\subsection{Orbit enumeration and the affine fundamental chamber}

We shall now explore in detail the orbit structure of $W$ on $Q / p Q$, in order to arrive at consequences of Conjecture 7.3.1 which can serve at least to test its plausibility. Our procedure will be to let $p$ vary arbitrarily, although eventually we will want $p$ relatively prime to $h$ for some of our conclusions.

Throughout we let $W$ be the Weyl group of a root system $\Phi$ in a Euclidean space $V$, with simple roots $\alpha_{1}, \ldots, \alpha_{\ell}$ and root lattice $Q=L(\Phi)$. The dual root system is $\Phi^{\vee}$, with simple coroots $\alpha_{1}^{\vee}, \ldots, \alpha_{\ell}^{\vee}$. The highest coroot we denote $\alpha_{0}^{\vee}$. The simple reflections (along the roots $\alpha_{i}$ ) are $s_{1}, \ldots, s_{l}$, and the reflection along the highest coroot is $s_{0}$. A subgroup of $W$ generated by a subset of $\left\{s_{0}, \ldots, s_{\ell}\right\}$ is called quasi-parabolic.

The affine weyl group $W_{a}$ is generated by $W$ and translations by elements of $Q$. (Note that this is dual to the formulation in [13].) The fundamental alcove is the open simplex

$$
A_{0}=\left\{\lambda \in V \mid\left(\alpha_{i}^{\vee}, \lambda\right)>0, \quad 1 \leq i \leq \ell ; \quad\left(\alpha_{0}^{\vee}, \lambda\right)<1\right\} .
$$

Its closure $\overline{A_{0}}$ is a fundamental domain for $W_{a}$ : every $W_{a}$ orbit meets $\overline{A_{0}}$ in a unique point.

Let us define $p W_{a}$ to be the subgroup of $W_{a}$ generated by $W$ and $p Q$. If $x \in Q$, then the $p W_{a}$ orbit of $x$ is the union of all $p Q$ cosets $w(x+p Q)$ for 
$w \in W$. Thus we have a natural correspondence between $W$ orbits in $Q / p Q$ in $p W_{a}$ orbits in $Q$.

In turn, every $p W_{a}$ orbit in $Q$ meets the simplex $p \overline{A_{0}}$ in a unique element of $Q$. Thus we have proved most of the following lemma.

LEMMA 7.4.1. There are natural bijective correspondences between the following three things:

(1) $W$ orbits in $Q / p Q$,

(2) $p W_{a}$ orbits in $Q$, and

(3) Elements of $Q \cap p \overline{A_{0}}$.

Furthermore, the stabilizer of an element of $Q / p Q$ represented by $x \in Q \cap p \overline{A_{0}}$ is the quasi-parabolic subgroup of $W$ generated by the reflections $s_{i}$ corresponding to walls of $p \overline{A_{0}}$ which contain $x$. In particular this stabilizer is trivial if and only if $x$ lies in the interior $p A_{0}$.

Proof. Only the "furthermore" still requires proof.

The statement is unchanged if we replace $p W_{a}$ by $W_{a}, p A_{0}$ by $A_{0}$, and the coset $x+p Q$ by $x+Q$, and do not require $x \in Q$. For simplicity we prove it in this form.

The stabilizer of $x$ in $W_{a}$ is generated by the reflections in those walls of $A_{0}$ which contain $x$. (This is a general theorem which applies to any reflection group, finite or infinite.)

Suppose $w \in W$ stabilizes $x+Q$. Thus $w x=y x$ for $y$ some translation in $Q$, so $y^{-1} w \in W_{a}$ fixes $x$. By the preceding observation, $y^{-1} w$ is a product of reflections in walls of $A_{0}$ which contain $x$.

The image of each such reflection under the natural map $W_{a} \rightarrow W=W_{a} / Q$ is the corresponding reflection $s_{i}$, while the image of $y^{-1} w$ is $w$. Hence $w$ belongs to the subgroup in question (and this subgroup clearly stabilizes $x+Q$ ).

By the lemma, each nonregular $W$ orbit in $Q / p Q$ is isomorphic to the permutation representation on cosets $W / R$ for a nontrivial reflection subgroup $R$. In particular, this implies that the sign representation does not occur for these orbits. Hence the multiplicity of the sign representation is the number of regular orbits in $Q / p Q$. If Conjecture 7.3.1 is to hold, this multiplicity must be 1 for $p=h+1$, since $R_{W}$ has only one invariant. Moreover, the total number of orbits in $Q /(h+1) Q$ must be the multiplicity of the sign representation in $R_{W}$.

We shall now derive formulas for the number of orbits and of regular orbits in $Q / p Q$, valid for certain $p$ including $p=h+1$. In this way we explain why for $p=h+1$ there is just one regular orbit, as there should be, and obtain a formula predicting the multiplicity of $\varepsilon$ in $R_{w}$.

Definition. A function $f(n)$ defined for $n \in \mathbb{N}$ is a quasi-polynomial with period 
$h$ if for each $i$ modulo $h$ there is a polynomial $p_{i}(n)$ such that $f(n)=p_{i}(n)$ for all $n \equiv i(\bmod h)$.

For a proof of the following theorem, see Stanley [23]. The function $e(P, n)$ is called the Ehrhart quasi-polynomial of $P$.

THEOREM 7.4.1. Let $P$ be a convex polytope in $\mathbb{R}^{\ell}$ whose vertices have rational coordinates. Let $e(P, n)=\left|n P \cap \mathbb{Z}^{\ell}\right|$ be the number of integer points in $n P$. Let $h$ be such that $h P$ has integer vertices. Then $e(P, n)$ is a quasi-polynomial with period $h$. Extending the definition of $e(P, n)$ to negative $n$ by quasi-polynomiality we have

$$
(-1)^{d} e(P,-n)=\bar{e}(P, n),
$$

where $\bar{e}(P, n)$ is the number of integer points in the interior of $n P$, and $d$ is the dimension of $P$.

Proposition 7.4.1. Fix a root system $\Phi$. The following table gives a period $h$ (not the best possible, in general) for the Ehrhart quasi-polynomial $e\left(\overline{A_{0}}, n\right)$, taking the integer points to be the root lattice.

\begin{tabular}{c|c}
$\Phi$ & $h$ \\
\hline$A_{\ell}$ & $\ell+1$ \\
$B_{\ell}$ & 4 \\
$C_{\ell}$ & 4 \\
$D_{\ell}$ & 8 \\
$E_{6}$ & 18 \\
$E_{7}$ & 24 \\
$E_{8}$ & 60 \\
$F_{4}$ & 12 \\
$G_{2}$ & 6
\end{tabular}

Proof. Let $a_{0}^{\vee}=c_{1} \alpha_{1}^{\vee}+\cdots+c_{\ell} \alpha_{\ell}^{\vee}$. Let $f$ be the connection constant (the index of the root lattice as a subgroup of the weight lattice). The vertices of $\overline{A_{0}}$ are the origin and the points $\lambda_{i} / c_{i}$ where $\lambda_{1}, \ldots, \lambda_{l}$ are the fundamental weights. In particular, if $c$ is the least common multiple of the $c_{i}$, then $c \overline{A_{0}}$ has vertices in the weight lattice. This implies that $c f \overline{A_{0}}$ has vertices in the root lattice and hence $c f$ is a period for $e\left(\overline{A_{0}}, n\right)$.

The table gives the value of $c f$ in each case. (See [13, p. 98] for a table of the values of $c_{i}$ and $f$ ). 
THEOREM 7.4.2. Fix a root system $\Phi$ with Weyl group $W$. Let $p$ be relatively prime to the period given in table (140). In particular, it suffices that $p$ be relatively prime to the Coxeter number $h$. Then the number of regular $W$ orbits in $Q / p Q$ is given by

$$
\frac{1}{|W|} \prod_{i=1}^{\ell}\left(p-e_{i}\right)
$$

and the total number of $W$ orbits by

$$
\frac{1}{|W|} \prod_{i=1}^{\ell}\left(p+e_{i}\right)
$$

where $e_{1}, \ldots, e_{\ell}$ are the exponents.

Proof. By Lemma 7.4.1, the number of orbits is $e\left(\overline{A_{0}}, p\right)$ and the number of regular orbits is $\bar{e}\left(\overline{A_{0}}, p\right)$. Using (139) we see that it suffices to prove either formula. Furthermore, since for $i$ relatively prime to a period $g$, there are infinitely many primes in the congruence class of $i$ modulo $g$, it suffices to prove the formula only for $p$ prime and sufficiently large.

The formula we choose to prove is (142). By Pólya's theorem the number of orbits is given by

$$
\frac{1}{|W|} \sum_{w \in W}|\operatorname{Fix}(w)|
$$

In characteristic 0 , we have the Shephard-Todd formula [21], [13, p. 63]

$$
\sum_{w \in W} t^{c(w)}=\prod_{i=1}^{\ell}\left(1+e_{i} t\right),
$$

where $c(w)$ is the codimension of the subspace fixed by $w$ in $V=\mathbb{R}^{\ell}$, that is, the rank of the matrix $T_{w}-I$, where $T_{w}$ is the matrix of $w$. Relative to the basis of simple roots, this is an integer matrix, and the rank of its reduction modulo $p$ is the same as its rank over $\mathbb{R}$ for all sufficiently large primes $p$. Thus for these primes we have $|\mathrm{Fix}(w)|=p^{\ell-c(w)}$. Setting $t=p^{-1}$ and multiplying $p^{\ell} /|W|$ in (144) we get the equality of (142) with (143).

For the claim about $p$ relatively prime to the Coxeter number, we have only to observe case by case that the Coxeter number is divisible by each prime factor of the period given by table (140).

Remark. What happens if we alternatively try to prove the formula (141) is quite interesting. The number of regular orbits is $1 /|W|$ times the number of points not on any reflecting hyperplane, modulo $p$. For $p$ sufficiently large all intersections of reflecting hyperplanes have the same dimension as in characteristic zero, and the number of points on such an intersection $F$ is $p^{\operatorname{dim}(F)}$.

By Möbius inversion on the lattice of intersections $\mathcal{L}$ we arrive at the formula 


$$
\frac{1}{|W|} \sum_{F \in \mathcal{L}} \mu(F, \mathbf{1}) p^{\operatorname{dim}(F)}
$$

for the number of regular orbits. The equality of this with (141) is an identity due to Orlik and Solomon [19]. Thus we see that via the combinatorial reciprocity theorem for Ehrhart polynomials we may derive either the Shephard-Todd or the Orlik-Solomon identity from the other.

Let us now evaluate (141) with $p=h+1$.

COROLLARY 7.4.1. There is exactly one regular $W$ orbit in $Q / p Q$ for $p=h+1$.

Proof. The exponents $e_{i}$ are symmetric with respect to the Coxeter number, i.e., the list of numbers $h-e_{i}$ reproduces the exponents again. Hence for $p=h+1$ formula (141) becomes $\Pi\left(1+e_{i}\right) /|W|=1$.

Remark. The symmetry of the exponents means that replacing $p$ by $p+h$ in (141) yields (142). Thus for $p$ relatively prime to $h$, the number of all root lattice points in $p \overline{A_{0}}$ equals the number of interior root lattice points in $(p+h) \overline{A_{0}}$.

Although it seems to have no direct bearing on Conjecture 7.3.1, there is another context in which the number $(h+1)^{\ell}$ arises in connection with the affine Weyl group $W_{a}$. Namely, for each root $\alpha$ let $H_{\alpha}$ and $J_{\alpha}$ be the two affine reflecting hyperplanes which are perpendicular to $\alpha$ and lie nearest to $A_{0}$ on either side. The complement of the union of all these hyperplanes falls into open convex regions. In the case of $A_{\ell}$, these regions are the connected components of the Kazhdan-Lusztig left cells for $W_{a}$. In every case, the number of them is $(h+1)^{\ell}$. See Shi [22] for these results and further details.

\subsection{Alfano-Reiner results for dihedral groups (all conjectures hold)}

J. Alfano and E. Reiner have determined exactly the Frobenius series of $R(m)=$ $R_{W}$ when $W=I_{2}(m)$, the dihedral group of order $2 m$. In a nutshell, they prove that the $\mathrm{sl}_{2}$ strings extending from degree $(0, k)$ to degree $(k, 0)$ for each $k$ account for everything, except for one copy of the sign representation in degree $(1,1)$.

Here we indicate how their results agree with extrapolations of the conjectures made in Section 7 for Weyl groups.

First of all, Conjectures 7.1.1 and 7.1.2 transfer directly to $I_{2}(m)$, which has rank $\ell=2$ and Coxeter number $h=m$. Thus the conjectures predict

$$
q^{m} \mathcal{H}_{R(m)}\left(q^{-1}, q\right)=\left(1+q+\cdots+q^{m}\right)^{2} .
$$


Alfano-Reiner gives

$$
\begin{aligned}
\mathcal{H}_{R(m)}\left(q^{-1}, q\right)= & 2+2 \sum_{i=1}^{m-1}\left(q^{-i}+q^{-i+2}+\cdots+q^{i-2}+q^{i}\right) \\
& +\left(q^{-m}+q^{-m+2}+\cdots+q^{m-2}+q^{m}\right),
\end{aligned}
$$

which is easily seen to match (146).

Conjecture 7.2.1 makes no sense if $W /=I_{2}(m)$ since there is no Lie algebra present. The operator conjecture does hold, however.

On its face, Conjecture 7.3.1 makes no sense for $W=I_{2}(m)$ either, but there is a reasonable way to extrapolate it. By the results of Section 7.4, in the Weyl group case the permutation representation on $Q / p Q$ is a sum of orbits of the form $1 \uparrow_{P}^{W}$, where $P$ is a quasi-parabolic subgroup. These orbits correspond to points of $Q$ in $p \overline{A_{0}}$.

When $m=3,4$, or 6 , so $I_{2}(m)$ is a Weyl group, $p \overline{A_{0}}$ is a triangle whose intersection with $Q$ consists of the following: (1) an interior point, giving a regular orbit $1 \uparrow_{1}^{W} ;(2)$ the origin, giving a trivial orbit $1_{W}$, and (3) $m$ points along the sides. For even $m$ ( 4 and 6 ) the $m$ points along the sides consist of $m / 2$ each along the sides corresponding to simple reflections $s_{1}$ and $s_{2}$, giving $m / 2$ orbits each of the form $1 \uparrow_{\left\langle a_{1}\right\rangle}^{W}$ and $1 \uparrow_{\left\langle z_{2}\right\rangle}^{W}$. For odd $m$ (that is, for $m=3$ ), there is a point along the side corresponding to $s_{0}$. Although the quasi-parabolic $\left\langle s_{0}\right\rangle$ has no meaning in $I_{2}(m)$ for general $m$, when $m$ is odd all reflections are conjugate, so all induced characters $1 \uparrow_{\langle s\rangle}^{W}$ are the same. The natural extrapolation of Conjecture 7.3.1 to $I_{2}(m)$, then, involves replacing $Q / p Q$ by the permutation representation

$$
\begin{aligned}
& 1_{W}+1 \uparrow_{1}^{W}+(m / 2)\left(1 \uparrow_{\left\langle s_{1}\right\rangle}^{W}+1 \uparrow_{\left\langle s_{2}\right\rangle}^{W}\right) \text { for } m \text { even, } \\
& 1_{W}+1 \uparrow_{1}^{W}+m \cdot 1 \uparrow_{\langle s\rangle}^{W} \text { for } m \text { odd. }
\end{aligned}
$$

Alfano and Reiner have verified that Conjecture 7.3.1 in this form agrees with their determination of the character of $R(m)$.

\section{Acknowledgment}

Research supported in part by NSF grant number DMS-9119355.

\section{Appendix: Frobenius and Hilbert series tables}

On the following pages are tables of the Hilbert series and Frobenius series of $R_{n}$, for $n$ up to 7 for the Hilbert series and 4 for the Frobenius series. The Hilbert series tables have the same format as Table (16). The Frobenius series tables are organized similarly, but instead of a number, the total dimension, each entry is now a formal sum of Ferrers diagrams, which stand for the corresponding Schur functions, or irreducible characters. 
$n=2$

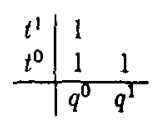

$n=3$

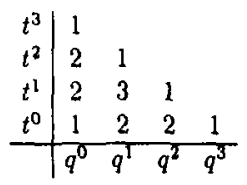

$n=4$

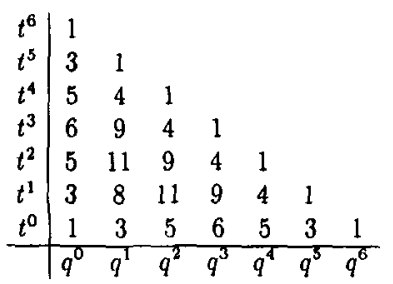

$n=5$

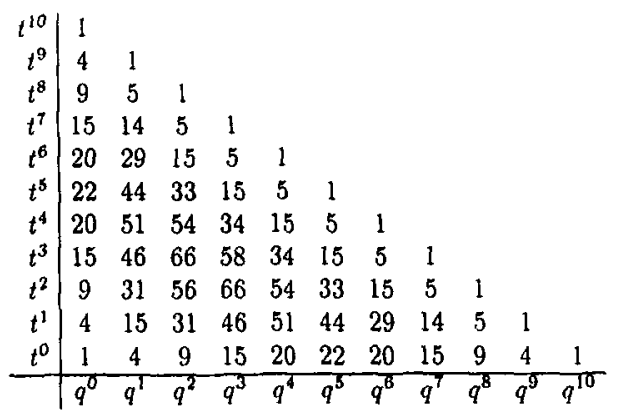




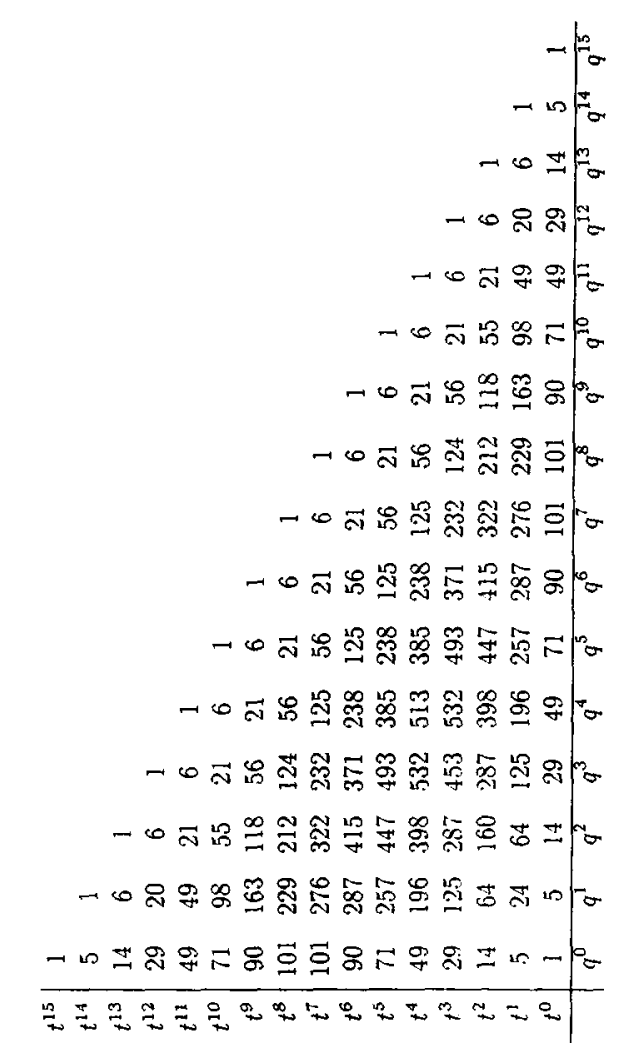

$\infty$ 


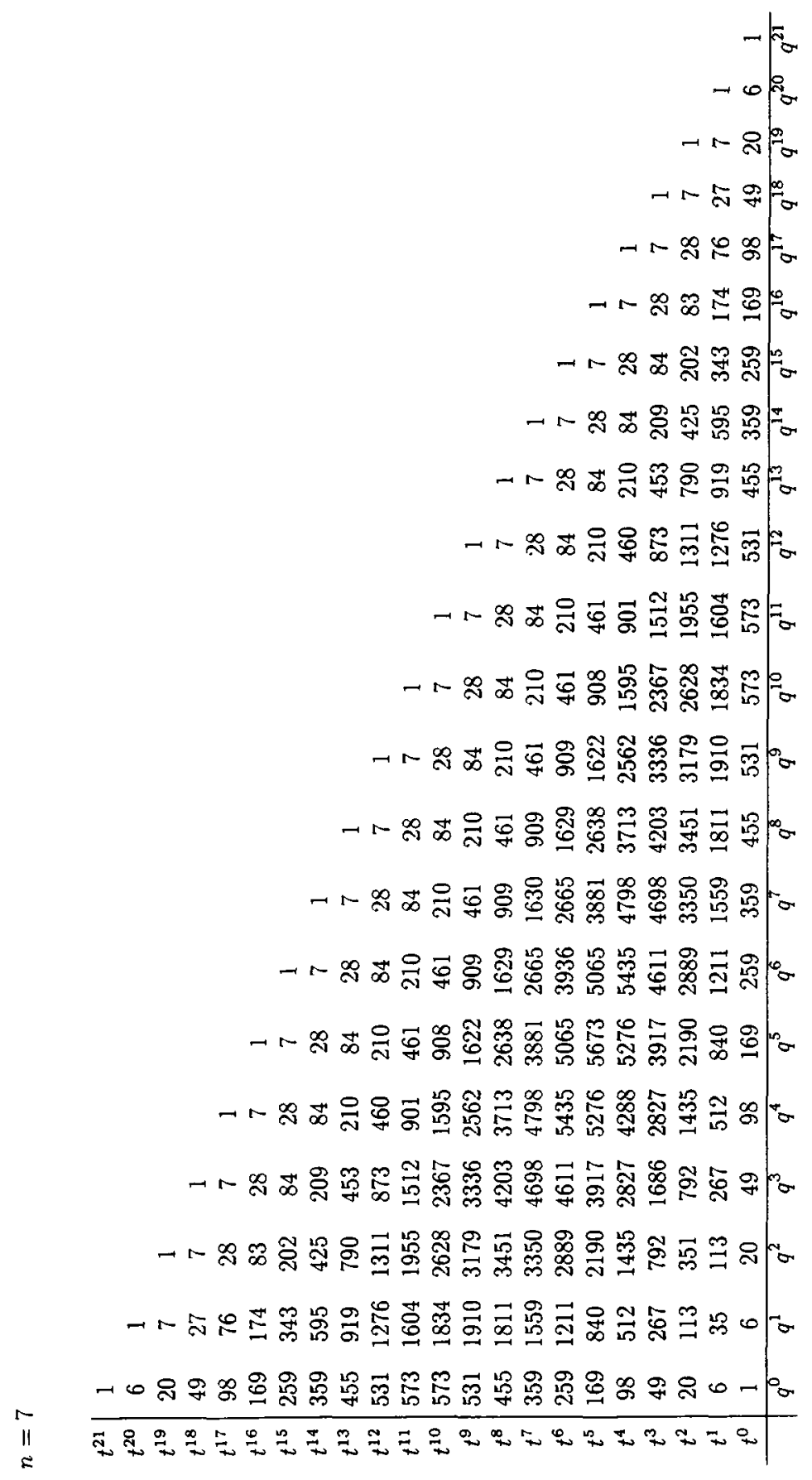



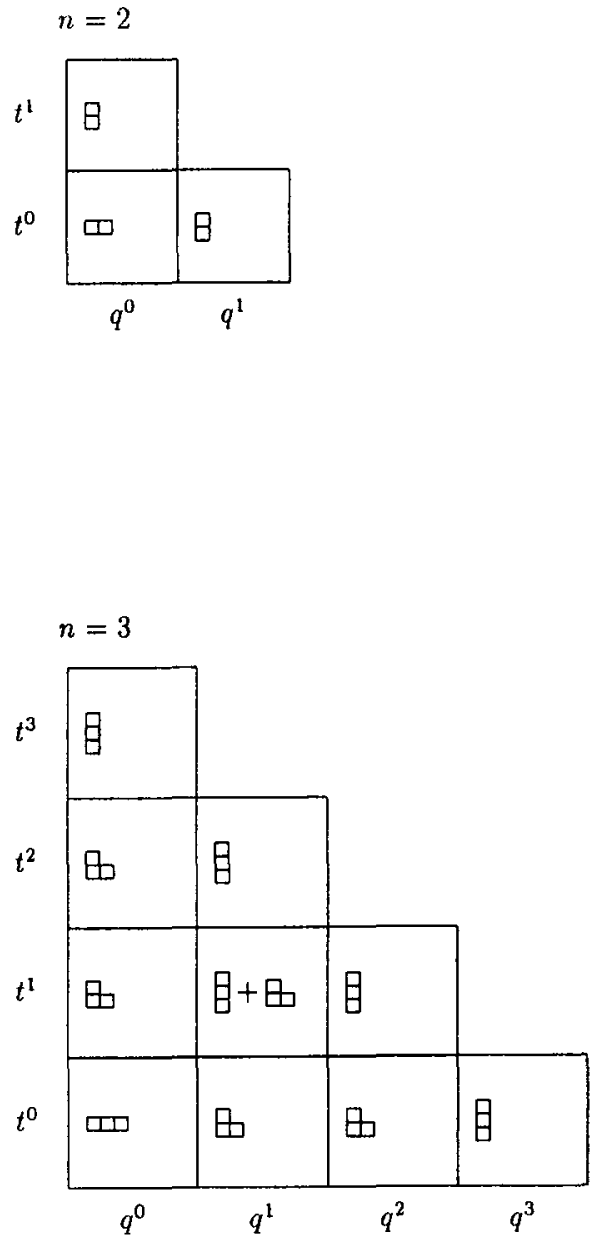


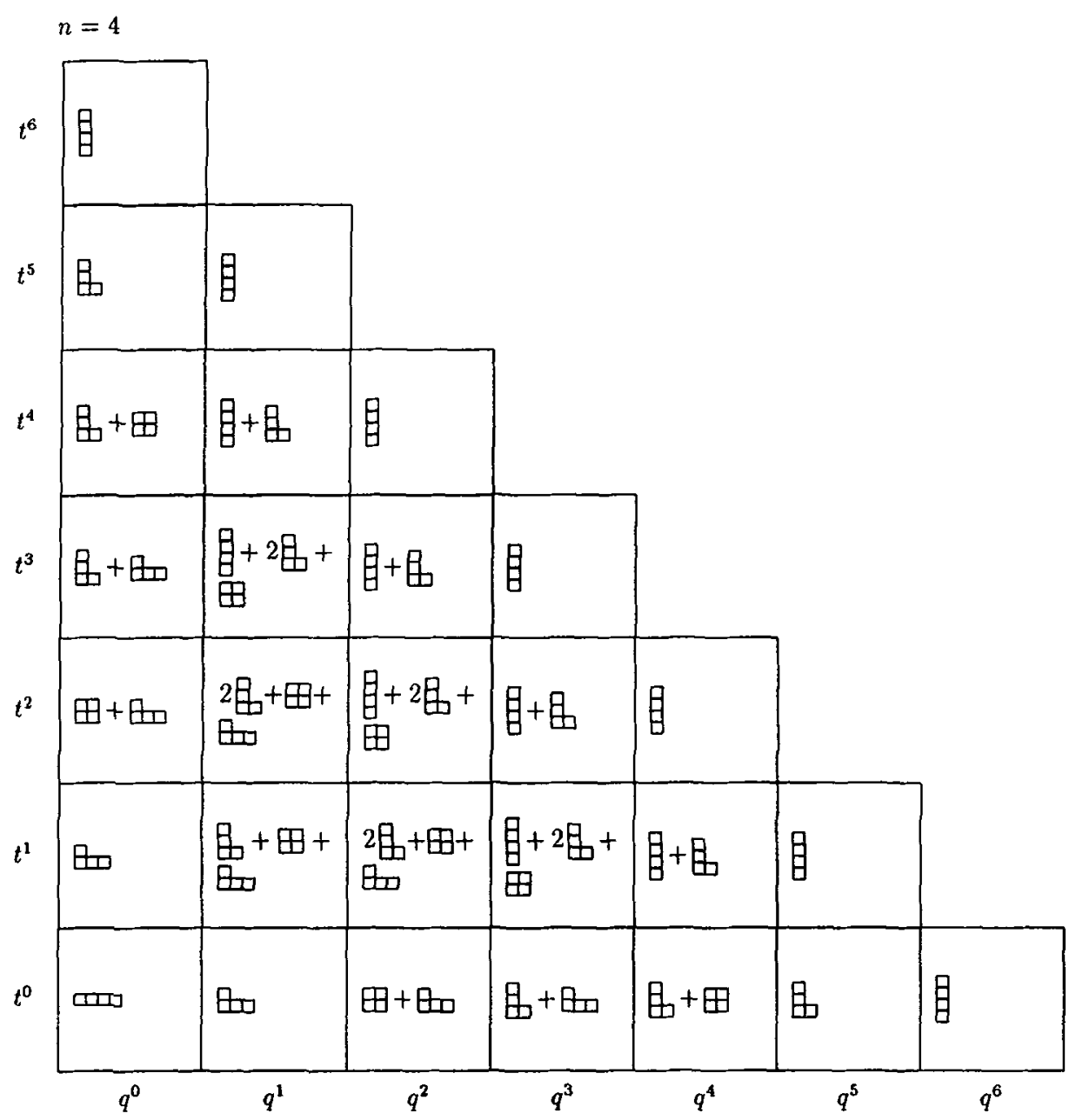




\section{References}

1. G.E. Andrews, "Identities in combinatorics III: A $q$-analog of the Lagrange inversion theorem," Proc. AMS 53 (1975), 240-245.

2. N. Bergeron and A.M. Garsia, "On certain spaces of harmonic polynomials," Contemporary Math. 138 (1992), 51-86.

3. L. Carlitz and J. Riordan, "Two element lattice permutation numbers and their q-generalization," Duke J. Math. 31 (1964), 371-388.

4. C. Chevalley, "Invariants of finite groups generated by reflections," Amer. J. Math. 77 (1955), 778-782.

5. J. Fürlinger and J. Hofbauer, " $q$-Catalan numbers," J. Comb. Theory $(A) 40$ (1985), 248-264.

6. A.M. Garsia, "A $q$-analogue of the Lagrange inversion formula," Houston J. Math. 7 (1981), 205-237.

7. A.M. Garsia and C. Procesi, "On certain graded $S_{n}$-modules and the $q$-Kostka polynomials," $A d v$. Math. 94 (1992), 82-138.

8. A.M. Garsia and J. Remmel, "A novel form of $q$-Lagrange inversion," Houston J. Math. 12 503-523.

9. M. Gerstenhaber, "On dominance and varieties of commuting matrices," Ann. Math. 73 (1961), 324-348.

10. I. Gessel, "A noncommutative generalization and $q$-analog of the Lagrange inversion formula," Trans. AMS 257 (1980), 455-482.

11. I. Gessel and D.-L. Wang, "Depth-first search as a combinatorial correspondence," J. Comb. Theory (A) 26 (1979), 308-313.

12. S. Helgason, Groups and Geometric Analysis: Integral Geometry, Invariant Differential Operators and Spherical Functions, Academic Press, New York, 1984.

13. J.E. Humphreys, Reflection Groups and Coxeter Groups, Cambridge University Press, Cambridge, England, 1990.

14. A.G. Konheim and B. Weiss, "An occupancy discipline and applications," SIAM J. Appl. Math. 14 (1966), 1266-1274.

15. B. Kostant, "Lie group representations on polynomial rings," Amer. J. Math. 85 (1963), 327-409.

16. G. Kreweras, "Une famille de polynômes ayant plusieurs propriétés énumeratives," Period. Math. Hungar. 11 (1980), 309-320.

17. I.G. Macdonald, Symmetric Functions and Hall Polynomials, Oxford University Press, Oxford, England, 1979.

18. C.L. Mallows and J. Riordan, "The inversion enumerator for labelled trees," Bull. AMS 74 (1968), 92-94.

19. P. Orlik and L. Solomon, "Unitary reflection groups and cohomology," Invent. Math. 59 (1980), 77-94.

20. R.W. Richardson, "Commuting varieties of semisimple Lie algebras and algebraic groups," Compositio Math. 38 (1979), 311-327.

21. G.C. Shephard and J.A. Todd, "Finite unitary reflection groups," Canad. J. Math. 6 (1954), 274-304.

22. J.-Y. Shi, "The Kazhdan-Lusztig cells in certain affine Weyl groups," Lecture Notes in Math 1179, Springer-Verlag, Berlin, 1986.

23. R.P. Stanley, Enumerative Combinatorics, Vol. I. Wadsworth \& Brooks/Cole, Monterey, CA, 1986.

24. R. Steinberg, "Finite reflection groups," Trans. $A M S 91$ (1959), 493-504.

25. R. Steinberg, "Invariants of finite reflection groups," Canad. J. Math. 12 (1960), 616-618.

26. R. Steinberg, "Differential equations invariant under finite reflection groups," Trans. AMS 112 (1964), 392-400.

27. W.T. Tutte, "A contribution to the theory of chromatic polynomials," Canad. J. Math. 6 (1953), $80-91$.

28. N. Wallach, "Invariant differential operators on a reductive Lie algebra and Weyl group representation," Manuscript, U.C.S.D. (1992).

29. H. Weyl, The Classical Groups, Their Invariants and Representations, Second Edition. Princeton University Press, Princeton, NJ, 1949. 